\title{
Analytic Studies of the Long Range Beam-Beam Tune Shifts and Chromaticities
}

\author{
Béla Erdélyi and Tanaji Sen \\ Beam Physics Department, MS 220 \\ Fermi National Accelerator Laboratory \\ Batavia, IL 60510
}

\begin{abstract}
A formula is derived, which allows efficient analytical evaluation of the long range beam-beam tune shifts and chromaticities with amplitude. It assumes that the beams are infinitely short, oppositely charged, and with Gaussian transversal profile. The formula employs an infinite sum with favorable convergence rates, making it well suited especially for the long range case. For a deeper understanding of the beam-beam effects, the structure of the formula was analyzed. It is shown that the tune shifts and chromaticities change sign at certain values of the separation and/or amplitude, and folds in the footprints are predicted. Some special cases were studied in more detail. Applications to the Tevatron are presented, including some proposed compensation schemes.
\end{abstract}

\section{Introduction}

Beam-beam interactions play a major role in circular colliders, as, for example, the Tevatron's Run II [1]. Tune shifts with amplitude are used to quantitatively characterize the strength of these interactions, which can be head-on, or long range. The amplitude (and parameter) dependent tune shifts to any order can be easily determined analytically from the map of a system using Differential Algebraic methods [2,3], if the potential has a good polynomial approximation (usually the Taylor expansion). The beam-beam potential between protons and antiprotons with Gaussian beam distributions is [4]:

$$
\begin{aligned}
U & =\frac{N_{b} r_{p}}{\gamma_{p}} \int_{0}^{\infty} \frac{d q}{\left[\left(2 \sigma_{x}^{2}+q\right)\left(2 \sigma_{y}^{2}+q\right)\right]^{1 / 2}} \\
& \times\left\{1-\exp \left[-\frac{\left(x_{\beta}+D_{x}\right)^{2}}{2 \sigma_{x}^{2}+q}-\frac{\left(y_{\beta}+D_{y}\right)^{2}}{2 \sigma_{y}^{2}+q}\right]\right\},
\end{aligned}
$$

where $N_{b}$ is the number of particles per bunch, $r_{p}$ is the classical proton radius, $\gamma_{p}$ is the relativistic factor, $\left(\sigma_{x}, \sigma_{y}\right)$ are the rms beam sizes in the two planes,$\left(x_{\beta}, y_{\beta}\right)$ are the betatron coordinates of a test particle and the separation between the two colliding bunches is $\vec{D}=\left(D_{x}, D_{y}\right)$ in the two planes respectively. This potential does not admit a rapidly converging polynomial expansion for amplitudes of practical interest. As a consequence of this form of the potential, computation of the amplitude dependent tune shift often requires tracking and subsequent postprocessing. Therefore, an analytical formula would be useful for the fast evaluation of the tune shifts, and would provide insight into the structure of the beam-beam effects. Moreover, an analytic formula for 
the computation of the amplitude dependent tune shifts can be readily modified to provide a useful tool for the determination of the amplitude dependent chromaticities.

The expression for head-on tune shift is well known [4], and an approximation for the long range tune shifts of round beams, which is valid in the large separation and small amplitude case, has been derived in [5]. In this note, we show that a formula can be derived for the tune shift that is always valid, and its evaluation is reduced to setting the truncation order in a reasonably fast converging infinite series, and a quadrature. All operations can be readily and quickly performed in, for example, Mathematica. The structure of the resulting formula as a function of amplitude and separation can be analyzed, providing asymptotic values for the sign changes of the tune shifts, and predicting folds in the tune footprint. In the next few sections, the derivations of the formulae are presented in some detail, and the theory is applied to the Tevatron in the last section. Also, some compensation schemes are proposed, and their effect on the dynamics is checked by tracking.

\section{Long Range Tune Shifts}

Technically, the amplitude dependent tune shift is the advance in angle along a torus in normal form space, where a particle moves with amplitude dependent frequency. Thus, the first step of the computation must be the transformation to normal form. In the Differential Algebraic picture, the transfer map is subjected to this transformation, while here, since the map is not easily computed, the transformation is applied directly to the Hamiltonian. Assuming a linearly dominated regime, it should be a very good approximation to make only a first order normal form transformation, and then take an average over the angles.

The first order normal form transformation to action-angle variables is achieved by the transformation $\left(x_{\beta}, x_{\beta}^{\prime} ; y_{\beta}, y_{\beta}^{\prime}\right) \mapsto\left(J_{x}, \phi_{x} ; J_{y}, \phi_{y}\right)$, with

$$
\begin{aligned}
& x_{\beta}=\sqrt{2 \beta_{x} J_{x}} \cos \phi_{x}, \\
& y_{\beta}=\sqrt{2 \beta_{y} J_{y}} \cos \phi_{y} .
\end{aligned}
$$

Assuming that the beam-beam interaction is the only perturbation to an otherwise simple harmonic motion with frequencies $\left(\nu_{x 0}, \nu_{y 0}\right)$, the Hamiltonian becomes

$$
H=\nu_{x 0} J_{x}+\nu_{y 0} J_{y}+U\left(J_{x}, \phi_{x} ; J_{y}, \phi_{y}\right) \delta\left(\theta-\theta_{c}\right),
$$

where $\delta(\theta)$ is the Dirac delta function, and $\theta$ is the independent variable. The delta function signifies that we neglect bunch length effects, and the interaction happens at a single collision point $\theta_{c}$. Introducing the tune shift as $\Delta \nu_{i}=$ $\nu_{i}-\nu_{i 0}$, where $i$ stands for $x$ or $y$, from Hamilton's equations of motion we obtain that the average change in phase advance is given by the following formula:

$$
2 \pi \Delta \nu_{i}=\frac{1}{(2 \pi)^{2}} \int_{0}^{2 \pi} \int_{0}^{2 \pi} \frac{\partial U\left(J_{x}, \phi_{x} ; J_{y}, \phi_{y}\right)}{\partial J_{i}} d \phi_{x} d \phi_{y} .
$$

The smoothness of the potential allows interchanging various integration and differentiation operations, and after performing the normal form transformation, (5) becomes (for $i=x$, and analogously for $y$ )

$$
\begin{aligned}
\Delta \nu_{x} & =C \int_{0}^{\infty} \frac{d q}{\left[\left(2 \sigma_{x}^{2}+q\right)\left(2 \sigma_{y}^{2}+q\right)\right]^{1 / 2}} \\
& \times\left[\frac{\partial}{\partial J_{x}}\left(e^{-p_{x}} I_{1}\left(J_{x}, D_{x}\right)\right)\right]\left[e^{-p_{y}} I_{2}\left(J_{y}, D_{y}\right)\right],
\end{aligned}
$$


where $C=-N_{b} r_{p} /\left((2 \pi)^{3} \gamma_{p}\right)<0$, and

$$
\begin{aligned}
& I_{1}\left(J_{x}, D_{x}\right)=\int_{0}^{2 \pi} \exp \left(-s_{x} \cos \phi_{x}+r_{x} \sin ^{2} \phi_{x}\right) d \phi_{x}, \\
& I_{2}\left(J_{y}, D_{y}\right)=\int_{0}^{2 \pi} \exp \left(-s_{y} \cos \phi_{y}+r_{y} \sin ^{2} \phi_{y}\right) d \phi_{y} .
\end{aligned}
$$

We introduced the following shorthand notations:

$$
p_{x}=\frac{2 \beta_{x} J_{x}+D_{x}^{2}}{2 \sigma_{x}^{2}+q}, \quad r_{x}=\frac{2 \beta_{x} J_{x}}{2 \sigma_{x}^{2}+q}, \quad s_{x}=\frac{2 D_{x} \sqrt{2 \beta_{x} J_{x}}}{2 \sigma_{x}^{2}+q} .
$$

Expanding the exponential in its Taylor series,

$$
e^{r_{x} \sin ^{2} \phi_{x}}=\sum_{k=0}^{\infty} \frac{r_{x}^{k} \sin ^{2 k} \phi_{x}}{k !},
$$

we obtain that

$$
I_{1}=\sum_{k=0}^{\infty} \frac{r_{x}^{k}}{k !} \int_{0}^{2 \pi} \exp \left(-s_{x} \cos \phi_{x}\right) \sin ^{2 k} \phi_{x} d \phi_{x} .
$$

If $s_{x} \neq 0$, a standard formula from the theory of Bessel functions [6] can be easily modified to give

$$
\int_{0}^{2 \pi} \exp \left(-s_{x} \cos \phi_{x}\right) \sin ^{2 k} \phi_{x} d \phi_{x}=2 \sqrt{\pi} \Gamma\left(k+\frac{1}{2}\right) I_{k}\left(s_{x}\right)\left(\frac{2}{s_{x}}\right)^{k} .
$$

At this point it should be noted that $s_{x}$ vanishes if $J_{x}$ or $D_{x}$ does, and, as a consequence, the final formula that will be obtained cannot be applied directly to these cases. However, it will be possible to obtain the zero amplitude tune shift values as limits. Here $I_{k}\left(s_{x}\right)$ is the modified Bessel function of the first kind, and $\Gamma\left(k+\frac{1}{2}\right)$ is the Gamma function.

The final result is useful because the sum (11) converges for any value of $r_{x}$ and $s_{x}$, and it converges reasonably fast. The sum is positive definite, since every term in the sum is positive; so the formula should be numerically stable. Using the ratio test to study convergence, we obtain

$$
R_{n}=\frac{2 r_{x}}{s_{x}} \frac{1}{n+1} \frac{\Gamma\left(n+\frac{3}{2}\right)}{\Gamma\left(n+\frac{1}{2}\right)} \frac{I_{n+1}\left(s_{x}\right)}{I_{n}\left(s_{x}\right)} .
$$

Using the recurrence relation for the Gamma function we obtain

$$
\frac{\Gamma\left(n+\frac{3}{2}\right)}{\Gamma\left(n+\frac{1}{2}\right)}=n+\frac{1}{2},
$$

and from the series expansion of the Bessel functions,

$$
I_{n}\left(s_{x}\right)=\sum_{t=0}^{\infty} \frac{\left(\frac{s_{x}}{2}\right)^{n+2 t}}{t ! \Gamma(n+t+1)},
$$

it follows that

$$
\frac{I_{n+1}\left(s_{x}\right)}{I_{n}\left(s_{x}\right)} \leq \frac{s_{x}}{2(n+1)} .
$$

Therefore, we obtain that

$$
R_{n} \leq r_{x} \frac{2 n+1}{2(n+1)^{2}}
$$


from which readily follows that the sum converges since

$$
\lim _{n \rightarrow \infty} R_{n}=0 .
$$

The value of the infinite sum depends on the separations $\left(D_{x}, D_{y}\right)$ but its rate of convergence does not. This is important, since it gives a measure of how many terms must be retained from the infinite sum. Comparing this ratio with the ratio test of the exponential function expansion of $r_{x}$,

$$
Q_{n}=\frac{r_{x}}{n+1},
$$

it can be seen that (11) converges faster than $e^{r_{x}}$, since $R_{n}<Q_{n}$, for any value of $n$. This is an important feature of the expansion which makes it suitable for numerical evaluation.

Furthermore, notice that the maximum value of $r_{x}$ is $J_{x} / \varepsilon_{x}$, where $\varepsilon_{x}=$ $\sigma_{x}^{2} / \beta_{x}$ is the emittance. It follows that in the infinite sum more terms need to be retained in case of large amplitudes. In (17) an approximate equality sign can be assumed in the small amplitude and separation case. Hence, for a fixed amplitude, at large separations $R_{n}$ is strictly smaller than $r_{x} \frac{2 n+1}{2(n+1)^{2}}$, giving better convergence in the long range case. That is why this form is well suited in the case of the parasitic collisions. From the expression for the potential it is clear that the tune shift vanishes at infinity.

Returning to the expression for the tune shift, we obtain that

$$
\Delta \nu_{x}=4 \pi C \int_{0}^{\infty} \frac{d q}{\left[\left(2 \sigma_{x}^{2}+q\right)\left(2 \sigma_{y}^{2}+q\right)\right]^{1 / 2}} \sum_{x} \sum_{y},
$$

where

$$
\begin{aligned}
& \sum_{x}=\sum_{k=0}^{\infty} \frac{\Gamma\left(k+\frac{1}{2}\right)}{k !} \frac{\partial}{\partial J_{x}}\left[e^{-p_{x}} I_{k}\left(s_{x}\right)\left(\frac{2 r_{x}}{s_{x}}\right)^{k}\right] \\
& \sum_{y}=\sum_{l=0}^{\infty} \frac{\Gamma\left(l+\frac{1}{2}\right)}{l !} e^{-p_{y}} I_{l}\left(s_{y}\right)\left(\frac{2 r_{y}}{s_{y}}\right)^{l} .
\end{aligned}
$$

The next step is to perform the partial differentiation in (21). The result is given by

$$
\begin{aligned}
\sum_{x} & =\sum_{k=0}^{\infty} \frac{\left(\frac{2 r_{x}}{s_{x}}\right)^{k}}{k !} \Gamma\left(k+\frac{1}{2}\right) \\
& \times\left[I_{k}\left(s_{x}\right)\left(\frac{2 k}{2 J_{x}}-\frac{2 \beta_{x}}{2 \sigma_{x}^{2}+q}\right)+I_{k+1}\left(s_{x}\right) \frac{s_{x}}{2 J_{x}}\right],
\end{aligned}
$$

where the following recurrence relation has been used [6]:

$$
s_{x} I_{k}^{\prime}\left(s_{x}\right)=k I_{k}\left(s_{x}\right)+s_{x} I_{k+1}\left(s_{x}\right) .
$$

The results can be expressed in a more elegant form if we change the integration variable from $q$ to $v$ by $v=1 /\left[1+q /\left(2 \sigma_{x}^{2}\right)\right]$, and introduce the ratio of rms beam sizes $r=\sigma_{y} / \sigma_{x}$. It is also convenient to introduce dimensionless variables for the amplitudes and separations according to

$$
a_{x}=\frac{\sqrt{2 \beta_{x} J_{x}}}{\sigma_{x}}, \quad d_{x}=\frac{D_{x}}{\sigma_{x}}
$$


and similarly define $a_{y}$ and $d_{y}$. Using these notations, we obtain the following relationships:

$$
\begin{aligned}
p_{x} & =\frac{v}{2}\left(a_{x}^{2}+d_{x}^{2}\right), & p_{y} & =f \frac{v}{2}\left(a_{y}^{2}+d_{y}^{2}\right), \\
r_{x} & =\frac{v}{2} a_{x}^{2}, & r_{y} & =f \frac{v}{2} a_{y}^{2}, \\
s_{x} & =v a_{x} d_{x}, & s_{y} & =f v a_{y} d_{y},
\end{aligned}
$$

where

$$
f=\frac{r^{2}}{v\left(r^{2}-1\right)+1} .
$$

Putting everything together, the final expression for the amplitude dependent tune shift in $x$ is

$$
\Delta \nu_{x}=\frac{4 \pi C}{\varepsilon_{x}} \int_{0}^{1} \frac{e^{-\left(p_{x}+p_{y}\right)}}{v\left[v\left(r^{2}-1\right)+1\right]^{1 / 2}} \sum_{x} \sum_{y} d v,
$$

where

$$
\begin{aligned}
\sum_{x} & =\sum_{k=0}^{\infty} \frac{\left(\frac{a_{x}}{d_{x}}\right)^{k}}{k !} \Gamma\left(k+\frac{1}{2}\right) \\
& \times\left[I_{k}\left(s_{x}\right)\left(\frac{2 k}{a_{x}^{2}}-v\right)+I_{k+1}\left(s_{x}\right) \frac{s_{x}}{a_{x}^{2}}\right], \\
\sum_{y} & =\sum_{l=0}^{\infty} \frac{\left(\frac{a_{y}}{d_{y}}\right)^{l}}{l !} \Gamma\left(l+\frac{1}{2}\right) I_{l}\left(s_{y}\right) .
\end{aligned}
$$

For completeness, the amplitude dependent tune shift in $y$ is similarly

$$
\Delta \nu_{y}=\frac{4 \pi C}{\varepsilon_{y}} \int_{0}^{1} \frac{e^{-\left(p_{x}+p_{y}\right)}}{v\left[v\left(r^{2}-1\right)+1\right]^{1 / 2}} \sum_{x}^{\prime} \sum_{y}^{\prime} d v,
$$

where

$$
\begin{aligned}
\sum_{x}^{\prime} & =\sum_{k=0}^{\infty} \frac{\left(\frac{a_{x}}{d_{x}}\right)^{k}}{k !} \Gamma\left(k+\frac{1}{2}\right) I_{k}\left(s_{x}\right), \\
\sum_{y}^{\prime} & =\sum_{l=0}^{\infty} \frac{\left(\frac{a_{y}}{d_{y}}\right)^{l}}{l !} \Gamma\left(l+\frac{1}{2}\right) \\
& \times\left[I_{l}\left(s_{y}\right)\left(\frac{2 l}{a_{y}^{2}}-f v\right)+I_{l+1}\left(s_{y}\right) \frac{s_{y}}{a_{y}^{2}}\right] .
\end{aligned}
$$

In Appendix A an alternate derivation is presented, and in a limiting case the connection with the method of this section is explored.

\section{The Sign of the Tune Shifts}

It is interesting to study the sign and extrema of the amplitude dependent tune shifts as a function of amplitude and separation. The understanding of this aspect may provide a correction scheme for the long range beam-beam interaction and help in the design of a separation scheme. It is sufficient to study the $x$ tune shift only; the $y$ can be done similarly. 
First, notice that from (6) it is clear that $\Delta \nu_{x}$ is positive definite for head-on interactions, $\vec{D}=0$, since it can be written as

$$
\begin{aligned}
\Delta \nu_{x} & =C \frac{\partial}{\partial J_{x}} \int_{0}^{\infty} \frac{d q}{\left[\left(2 \sigma_{x}^{2}+q\right)\left(2 \sigma_{y}^{2}+q\right)\right]^{1 / 2}} \\
& \times\left[\left(e^{-p_{x}} I_{1}\left(J_{x}, 0\right)\right)\right]\left[e^{-p_{y}} I_{2}\left(J_{y}, 0\right)\right],
\end{aligned}
$$

and the integral is a positive definite decreasing function of $J_{x}$. Hence, its derivative with respect to $J_{x}$ is negative definite. Combining this fact together with $C<0$ implies that the tune shift is always positive for head-on collision of oppositely charged beams. For non-zero separations, the vanishing amplitude case can be more easily studied. This is done in the next subsection, followed by the case of non-zero amplitudes. It is also clear from (28), (29), and (30) that the tune shift depends only on the absolute value of the separations in both planes, so without loss of generality the separations are taken to be positive.

\subsection{Long Range Tune Shifts at Vanishing Amplitude}

The vanishing amplitude case can be studied fairly easily. The following results are obtained from (28) by taking analytical limits:

$$
\lim _{\substack{a_{x} \rightarrow 0 \\ a_{y} \rightarrow 0}} \Delta \nu_{x}=\xi \int_{0}^{1} \frac{e^{-\frac{d_{x}^{2}+f d_{y}^{2}}{2}} v}{\sqrt{v\left(r^{2}-1\right)+1}}\left(1-d_{x}^{2} v\right) d v,
$$

where

$$
\xi=\frac{N_{b} r_{p}}{4 \pi \gamma_{p} \varepsilon_{x}}>0
$$

is the beam-beam parameter. In the round beam case, $r=1$, the integral can be done analytically to give

$$
\lim _{\substack{a_{x} \rightarrow 0 \\ a_{y} \rightarrow 0}} \Delta \nu_{x}(r=1)=\xi \frac{2}{d^{4}}\left\{e^{-\frac{d^{2}}{2}}\left[d_{x}^{2} d^{2}+\left(d_{x}^{2}-d_{y}^{2}\right)\right]-\left(d_{x}^{2}-d_{y}^{2}\right)\right\} .
$$

where $d^{2}=d_{x}^{2}+d_{y}^{2}$. It is apparent from (37) that the loci of vanishing tune shifts in the case of large enough separations are in the close proximity of the diagonal in the $\left(d_{x}, d_{y}\right)$ plane (or, equivalently $\theta=\pi / 4$ in the polar coordinates $\left.d_{x}=d \cos \theta, d_{y}=d \sin \theta\right)$, since the first term in the curly brackets is suppressed already at modest separations and the second term vanishes along the diagonal. This is confirmed by an accurate numerical solution; the exact dependence of $\Delta \nu_{x}$ on $d_{x}$ and $d_{y}$ for a few representative samples of vanishing amplitudes and different aspect ratios is shown in Figures 1 and 2. Notice that the tune shifts essentially depend on $\left|d_{x}\right|$ only if $r \ll 1$, and on $d$ if $r \gg 1$. Figure 3 shows the well-known projections of Figure 1 in the round case into the $\left(d_{x}, \Delta \nu_{x} / \xi\right)$ and $\left(d_{y}, \Delta \nu_{x} / \xi\right)$ planes for $d_{y}=0$ and $d_{x}=0$, respectively. 

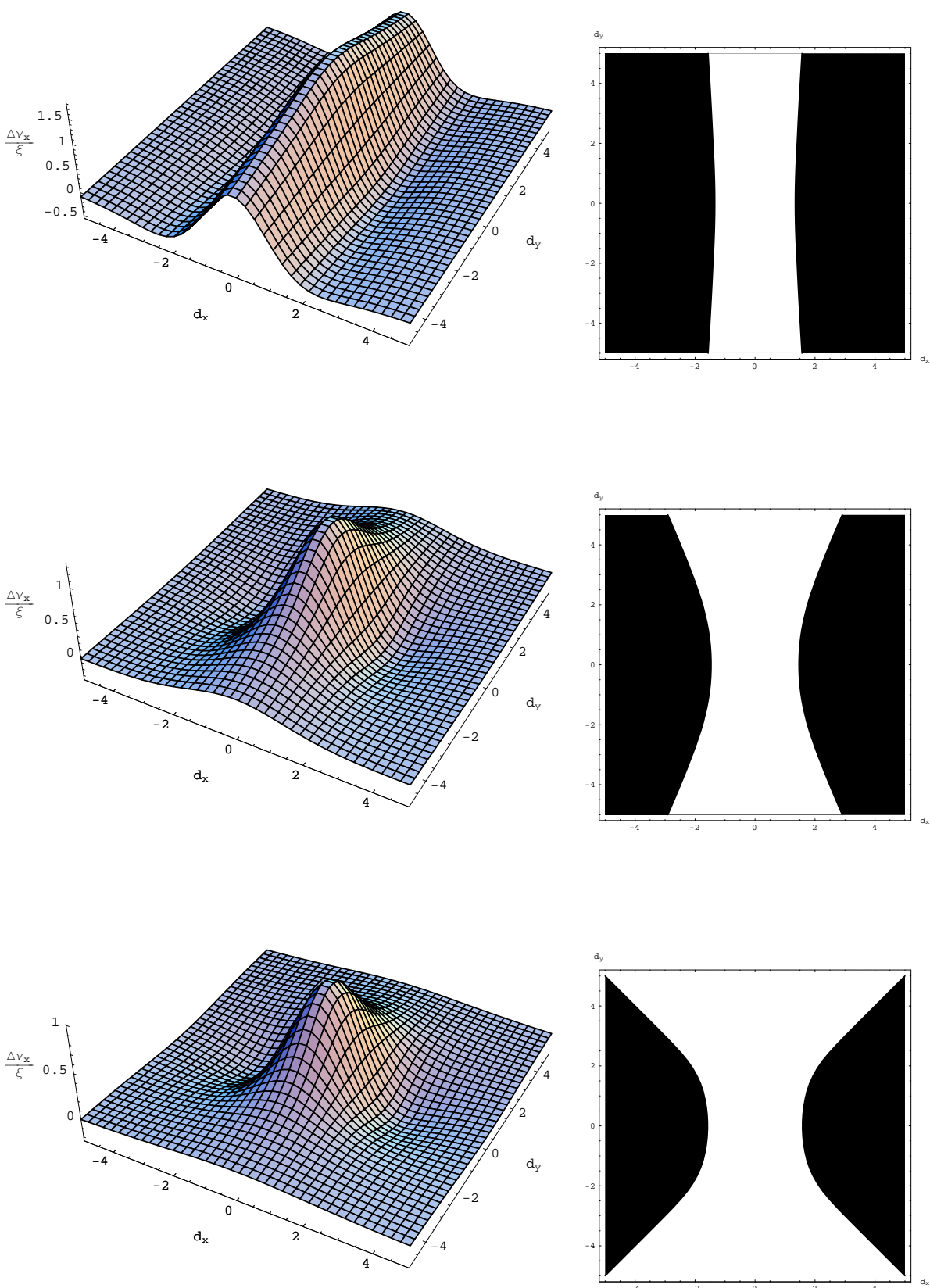

Figure 1: Horizontal tune shift dependence on the separations at zero amplitudes in the case of the following aspect ratios: $r=0.1, r=0.5$, and $r=1$ (round beam). In the contour plots the tune shift vanishes on the border between the dark and light regions. The dark areas correspond to negative values, while the light area to positive values. 

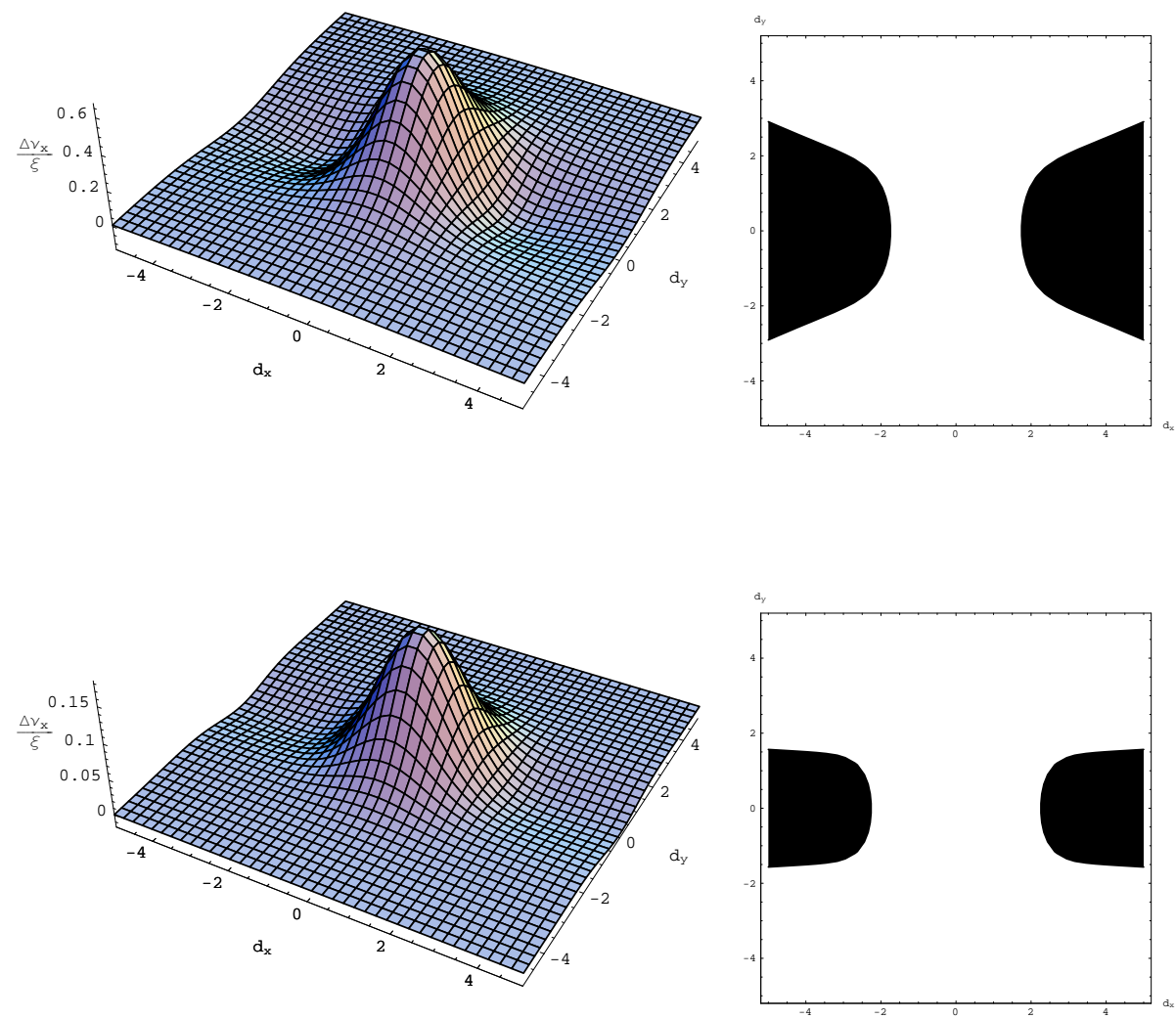

Figure 2: Continuation of Figure 1 for the following aspect ratios: $r=2$ and $r=10$.
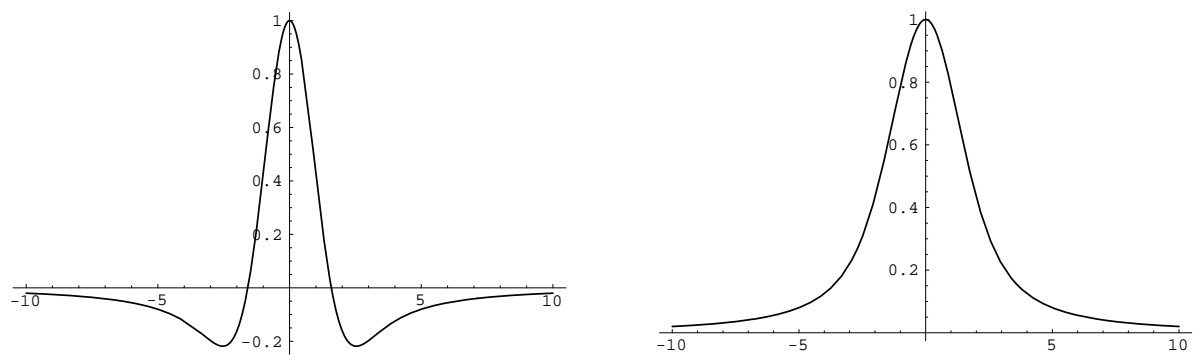

Figure 3: The zero amplitude horizontal tune shift in the case of a round beam, as a function of $\left(d_{x}, 0\right)$ and $\left(0, d_{y}\right)$, respectively. 
The location closest to the origin where $\Delta \nu_{x}$ changes sign is at $\left|d_{x}\right| \approx 1.5852$ for $d_{y}=0$; this is also the location of the maximum of the beam-beam force. It is also worthwhile to note that if $r>1$ then the maximum of the long-range tuneshift $\max \Delta \nu_{x}<\xi$, and if $r<1$ then $\max \Delta \nu_{x}>\xi$.

Variations in the emittance occur naturally from bunch to bunch in a train of bunches and it is also possible to control the average emittance to some extent. Hence the scaling of the long range beam-beam effects with the emittance is of interest. The Tevatron is currently operating with beams whose emittances are about $25 \%$ larger than design values. Assuming that the two transverse emittances are equal $\varepsilon=\varepsilon_{x}=\varepsilon_{y}$, the zero amplitude tune shift for round beams is of the form

$$
\lim _{\substack{a_{x} \rightarrow 0 \\ a_{y} \rightarrow 0}} \Delta \nu_{x}(r=1) \propto a\left[e^{-\frac{b}{\varepsilon}}\left(\frac{c}{\varepsilon}+1\right)-1\right],
$$

where $a, b, c$ are constants for fixed physical separations and beta functions. Therefore, in the limit of the emittance going to zero, the tune shift tends to a constant value, which in general is small but non-zero. An exception is when $D_{x}^{2} / \beta_{x} \approx D_{y}^{2} / \beta_{y}$, in which case

$$
\lim _{\substack{a_{x} \rightarrow 0 \\ a_{y} \rightarrow 0}} \Delta \nu_{x}(r=1) \propto \mathrm{const} \frac{e^{-\frac{b}{\varepsilon}}}{\varepsilon},
$$

and in the limit $\varepsilon \rightarrow 0$ the tune shift vanishes. In the limit of the emittance going to infinity the tune shift goes to zero in general. Therefore, the absolute value of the tune shift must have a maximum as a function of the emittance. It is straightforward to check that the maximum occurs at

$$
\varepsilon_{\max }=\frac{D_{x}^{2}}{\beta_{x}} \quad \Rightarrow D_{x}^{2}=\sigma_{x}^{2} \quad \text { or } \quad d_{x}=1
$$

and the tune shift decreases slowly for $\varepsilon>\varepsilon_{\max }$ and fast for $\varepsilon<\varepsilon_{\max }$, as shown in Figure 4. Therefore, according to (40), the situation when the separation equals the beam size might be dangerous.

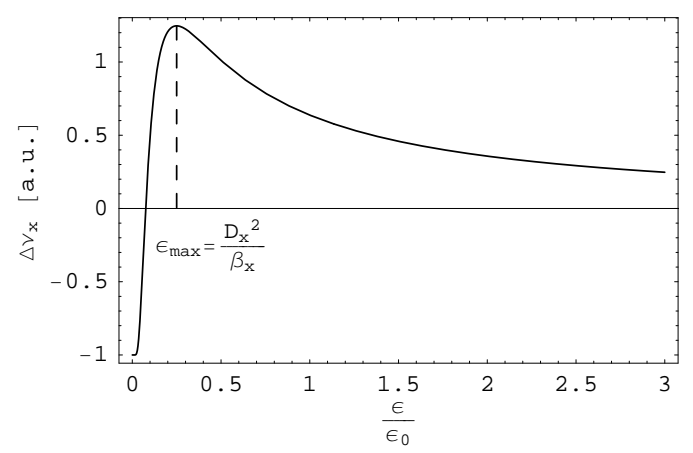

Figure 4: Qualitative behavior of the zero amplitude, round beam, horizontal tune shift with long-range interactions as a function of emittance, assuming equal horizontal and vertical emittances. The physical separations $D_{x}, D_{y}$ are kept constant. Note that the scaling is qualitatively different from that in the head-on case where $\Delta \nu_{x} \propto 1 / \epsilon$.

The integral (35) cannot be done analytically if $r \neq 1$. The question arises whether the loci of vanishing tune shift will still be in the proximity of $\theta=\pi / 4$. Without presenting algebraic details, expanding the angle and aspect ratio to 

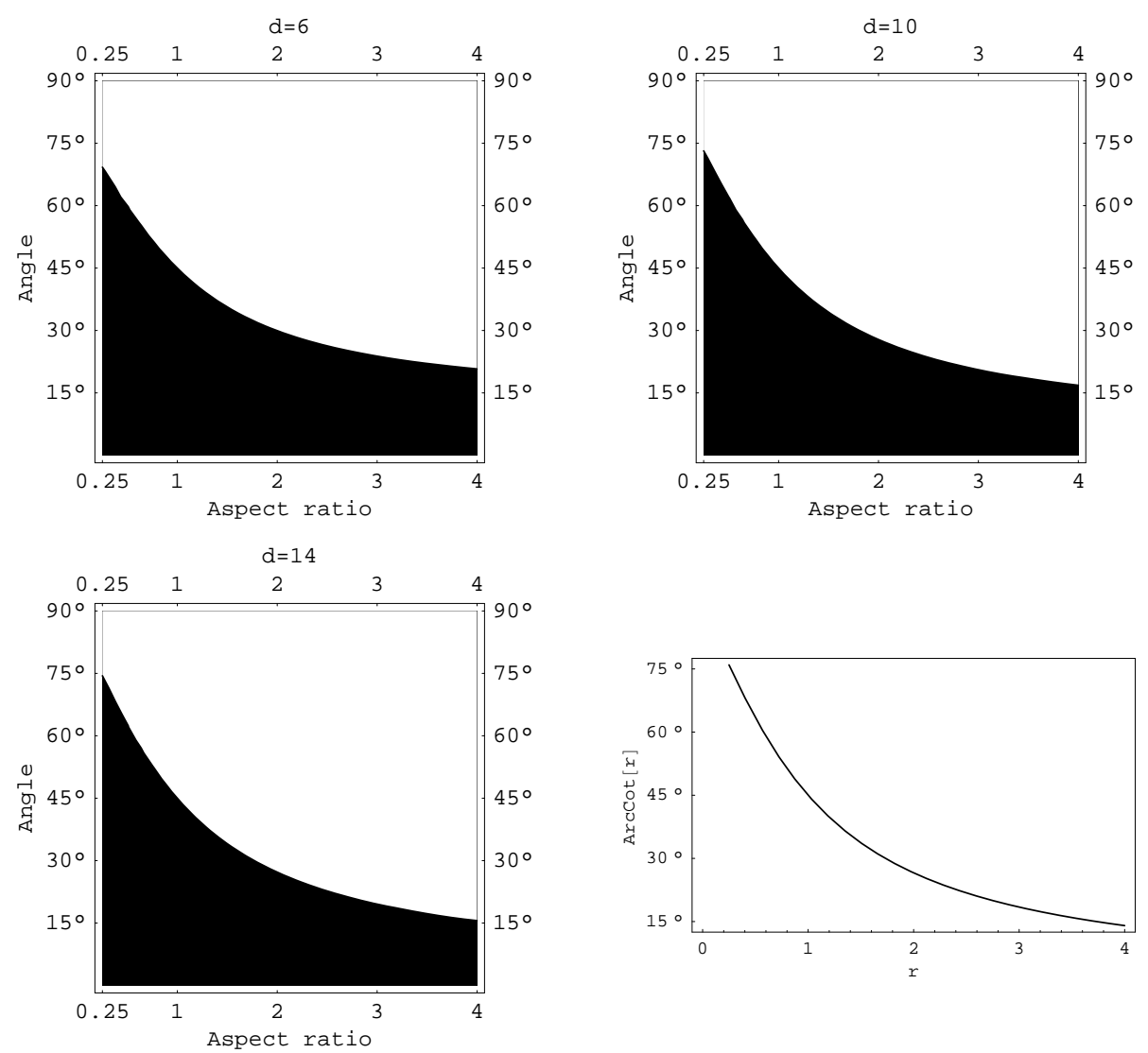

Figure 5: Contour of vanishing tune shifts for the following case: zero amplitudes, the separations are: a) $d=6$ (minimum separation in the Tevatron), b) $d=10$ (average separation in the Tevatron), c) $d=14$ (maximum separation in the Tevatron), and the contour represents the polar angle at which the tune shifts vanish for aspect ratios in the range $[0.25,4]$. Also shown is d) the function $\operatorname{arccot}(r)$, to which the contours apparently converge in the large separation limit.

first order according to $\theta_{\min }=\pi / 4+\delta \theta$ and $r=1+\delta r$, the integral can be evaluated to give for the loci of vanishing tune shifts

$$
\delta \theta=\frac{d^{2}}{2\left(2+d^{2}-2 e^{\frac{d^{2}}{2}}\right)}+\frac{\delta r}{2}\left(\frac{2 d^{2}}{2+d-2 e^{\frac{d^{2}}{2}}}+\frac{6}{d^{2}}-1\right) .
$$

As previously shown, if $\delta r=0$, the deviation of $\theta_{\min }$ from $\pi / 4$ is negligibly small even for modest separations. In the limit of large separations we obtain that

$$
\lim _{d \rightarrow \infty} \theta_{\min }(\delta r)=\frac{\pi}{4}-\frac{\delta r}{2} .
$$

Thus the locations of vanishing tune shift do depend on the beam aspect ratio. This approximation breaks down if $\delta r$ is not small or if $d$ is not large.

The exact dependence can be computed by numerical integration. A useful insight is obtained by inspecting the figures obtained using separations in the range $6 \leq d \leq 14$, and aspect ratios in the interval $r \in[0.25,4]$. This is the Tevatron's case, the average separation being $d \approx 10$. The simple estimate (41), valid for $d=10$ and around $r=1$, gives $\theta_{\min }(r)=\pi / 4-0.47(r-1)$. The 
global picture is revealed by the contours along which the long range tune shift vanish, as shown in Figure 5. Clearly, the contours tend to the graph of

$$
\theta_{\min }=\operatorname{arccot}(r)
$$

in the limit of large separation, and show only a weak dependence on $d$ in the range relevant for the Tevatron. Although it is not obvious analytically from (35) why this happens, it has a simple intuitive interpretation. Notice that in this case

$$
\frac{D_{x}}{D_{y}}=\frac{d_{x}}{d_{y} r}=\frac{\cot \left(\theta_{\min }\right)}{r}=1 .
$$

Therefore, it has the meaning of choosing equal physical separations for any aspect ratio, if the separations are large. It can be understood if one considers that from large distances the strong beam is point-like, regardless of its actual aspect ratio, so the tune shift cancellation has the same symmetry as in the case of round beams.

In the following we try to understand the behavior of the tune shifts at non-zero amplitudes.

\subsection{Long Range Tune Shifts at Non-Zero Amplitudes}

Although the amplitude dependent tune shift is given by a doubly infinite series, it can be shown that its sign is determined by the first two terms in the expansions. Indeed, in the case of vanishing amplitudes only the first two terms of the expansion survive the limit $a_{x} \rightarrow 0, a_{y} \rightarrow 0$. In the case of non-zero amplitudes the argument is based on analyzing the structure of (29).

Recall that the head-on tune shifts are positive at any amplitude. Thus, one expects that this result persists by continuity, at least for sufficiently small separations. Inspection of (28), (29), and (30) reveals that there is only one term in the sums that is not positive, and thus must be the dominating one in the small separation case. We observe that the term $2 k / a_{x}^{2}-v$, appearing in (29), is always positive for sufficiently large $k$, that is for $k>a_{x}^{2} / 2$. This shows that the high enough order contributions for off-center beam-beam collisions are always defocusing. Clearly, this threshold $k=k_{t}$ is actually lower than $a_{x}^{2} / 2$ due to the second term in (29), which is always positive, and the integration over $v$. Moreover, once the threshold $k_{t}$ is reached (where the terms in (29) become positive for $k>k_{t}$ ), terms with $k>k_{t}$ cannot change sign anymore. It follows that if $k_{t}=0$, the sign of the term with $k=0$ determines the sign of the tune shift.

On the other hand, if $k_{t}>0$, the sign still will be given by the terms that contribute to the vanishing amplitude case, namely

$$
E=-I_{0}\left(s_{x}\right) v+I_{1}\left(s_{x}\right)\left(\frac{s_{x}}{a_{x}^{2}}+\frac{v}{s_{x}}\right) .
$$

The other terms in the expansion tend to zero for vanishing amplitudes. To see that this is true, first consider the small amplitude case. As shown in the preceding section, by (17) the convergence must be really fast in this case, so the higher order contributions cannot change the sign of the tune shift. As the amplitude increases, so does $k_{t}$, hence the contributions with positive sign come from terms with high values of $k$ when again the corrections are tiny, and cannot change the tune shift's sign anymore. In summary, if $k_{t}>0$, the worst case is for $k_{t}=2$, but this corresponds to small amplitudes, and the fast convergence of the series prevents significant changes to the tune shift. 


\subsubsection{The small separation case}

Based on the previous reasoning, one expects that for $d_{x}$ sufficiently small, the terms with $k=0,1$ can never be positive. To check this, we need to estimate

$$
T=\int_{0}^{1} \frac{e^{-\left(p_{x}+p_{y}\right)} \sum_{y}}{v\left[v\left(r^{2}-1\right)+1\right]^{1 / 2}} E d v
$$

Utilizing again the formula

$$
I_{1}\left(s_{x}\right) \leq I_{0}\left(s_{x}\right) \frac{s_{x}}{2}
$$

we obtain that

$$
E<\frac{I_{0}\left(s_{x}\right) v}{2}\left(d_{x}^{2} v-1\right) .
$$

The integrand in $T$ is a continuous function of $v$ at every point except zero, i.e. for $v \in(0,1]$. Therefore, if we denote the integrand by $\lambda(v)$, we can split $T$ according to

$$
T=\lim _{v \rightarrow 0} \lambda(v)+\int_{\varepsilon}^{1} \lambda(v) d v
$$

for any $\varepsilon>0$. It is easy to see that

$$
\lim _{v \rightarrow 0} \lambda(v)=-\frac{\sqrt{\pi}}{2}
$$

Applying the integral form of the mean value theorem to the second part of the right hand side of (49), we obtain that

$$
T<-\frac{\sqrt{\pi}}{2}+\left(\frac{e^{-\left(p_{x}+p_{y}\right)} \sum_{y}}{\left[v\left(r^{2}-1\right)+1\right]^{1 / 2}} \frac{I_{0}\left(s_{x}\right)}{2}\left(d_{x}^{2} v-1\right)\right)_{v=v_{m}},
$$

for some $v_{m} \in(\varepsilon, 1)$, or equivalently

$$
T<-\frac{\sqrt{\pi}}{2}+K_{1}\left(d_{x}^{2} v_{m}-1\right),
$$

where $K_{1}$ is a positive number that depends on the amplitudes, separations, and beam aspect ratio. In fact, it can be shown that $v_{m}<1 / 2$, so it follows that $T<0$ whenever $\left|d_{x}\right|<\sqrt{2}$, that is

$$
\Delta \nu_{x}>0 \text { if }\left|d_{x}\right| \lesssim \sqrt{2} .
$$

Depending on the exact values of $K_{1}$ and $v_{m}, \Delta \nu_{x}$ may stay positive even if $\left|d_{x}\right|$ exceeds $\sqrt{2}$ (as previously shown, in the vanishing amplitude and vertical separation case for round beams it is actually 1.5852). Therefore, the first conclusion can be drawn: $\Delta \nu_{x}>0$ if $\left|d_{x}\right| \lesssim \sqrt{2}$, independent of the amplitudes and the separation in the $y$ plane, but in some specific cases the cutoff value for $\left|d_{x}\right|$ could be somewhat larger. However, according to (37), this conclusion must break down if $\left|d_{x}\right|$ is sufficiently large and $a_{x}$ is small.

\subsubsection{The large separation case}

At the other end of the spectrum, i.e. large separations and amplitudes, we can use the asymptotic relation $[6]$

$$
I_{0}\left(s_{x}\right) \approx I_{1}\left(s_{x}\right) .
$$


In this case we obtain that

$$
E \approx \frac{I_{0}\left(s_{x}\right) v}{a_{x}}\left(d_{x}+\frac{1}{d_{x}^{2} v}-a_{x}\right),
$$

and for the integral that

$$
T \approx-\frac{\sqrt{\pi}}{2}+\left(\frac{e^{-\left(p_{x}+p_{y}\right)} \sum_{y}}{\left[v\left(r^{2}-1\right)+1\right]^{1 / 2}} \frac{I_{0}\left(s_{x}\right)}{a_{x}}\left(d_{x}+\frac{1}{d_{x} v}-a_{x}\right)\right)_{v=v_{m}}
$$

or equivalently

$$
T \approx-\frac{\sqrt{\pi}}{2}+K_{2}\left(d_{x}+\frac{1}{d_{x} v_{m}}-a_{x}\right),
$$

where $K_{2}$ is a positive number. It follows that $T<0$ if $d_{x}+1 /\left(d_{x} v_{m}\right)-a_{x}<0$, that is if

$$
a_{x}>d_{x}+\frac{1}{d_{x} v_{m}} .
$$

A close inspection of the range of validity of (54) reveals that the correction for intermediate values of the separation is to slightly increase the right hand side of (58). Therefore, we can conclude that for large separations

$$
\Delta \nu_{x}>0 \text { if } a_{x} \gtrsim\left|d_{x}\right| .
$$

It is interesting to study the case $a_{x}<d_{x}$ for large separations. The sign of the tune shift in this case will depend on the specific value of $K_{2}$. We already proved that for a fixed large horizontal separation the tune shift is negative for vanishing horizontal amplitude, and positive at large horizontal amplitudes. This means that the tune shift must change sign at some intermediate horizontal amplitude. The location of sign change depends on $K_{1}$ and $K_{2}$, that is the vertical separation and amplitude, and beam aspect ratio. What can be inferred is that, for a given value for $\left|d_{x}\right|$ and $a_{x}$, which give negative tune shift at zero vertical amplitude and separation, the values of both $K_{1}$ and $K_{2}$ tend to decrease (since to first order they scale like $e^{-\left(a_{y}^{2}+d_{y}^{2}\right) / 2} I_{0}\left(a_{y} d_{y}\right)$, and have a maximum at $\left.a_{y}=\left|d_{y}\right|\right)$ as the vertical amplitude and/or separation increases, making it harder for the tune shift to stay negative.

\subsubsection{Summary}

It is reasonable to expect that the transition in the behavior of the tune shift from small to large separations happens smoothly, and one can extrapolate the asymptotic results to the following qualitative statement for the horizontal tune shift:

For small enough horizontal separation the beam-beam effect is focusing at any amplitude and vertical separation. Above a certain threshold of the horizontal separation, the beam-beam interaction becomes defocusing for particles with small amplitudes and vertical separation, and focusing for particles with large horizontal amplitudes independent of the vertical separation and amplitude. The exact location of the sign change depends on the vertical separation and amplitude, and beam aspect ratio.

Therefore, for given (sufficiently large) separations and given beam aspect ratio there is a boundary in the action space $\left(a_{x}, a_{y}\right)$ where the tune shifts must vanish, and the particles inside the boundary (smaller amplitudes) will 
have dynamically different behavior than outside the core (large amplitudes). Moreover, since the tune shift scales like the derivative of $e^{-\left(a_{x}^{2}+d_{x}^{2}\right) / 2} I_{0}\left(a_{x} d_{x}\right)$ w.r.t. $a_{x}$, the tune shift starts at a negative value at zero amplitude, might have a minimum negative value, then cross zero and have a maximum positive value before decaying to zero at infinite amplitudes. This may result in folds in the tune footprint, and could result in significant resonance widths around the folds' locations. Another type of folding may occur around the maximum value of the function $e^{-\left(a_{y}^{2}+d_{y}^{2}\right) / 2} I_{0}\left(a_{y} d_{y}\right)$ independent of the horizontal amplitude and separation. The locations of these folds are independent of the tune. If the tunes are such that the resonances occur at the folds, then we may have large resonance widths because $\Delta J_{\text {res }} \propto 1 / \partial(\Delta \nu) / \partial J$.

Another interesting aspect is that the same value of $a_{x}$ gives the location of vanishing horizontal tune shift and the critical point of the expression that appears in the vertical tune shift (31). Being the only critical point of a positive definite function that decays to zero at infinity, it follows that the vertical tune shift will attain its maximum value at the location of vanishing horizontal tune. This is also true if $x$ and $y$ are interchanged.

For a better quantitative understanding, we computed the horizontal tune shifts as a function of the amplitudes $\left(a_{x}, a_{y}\right)$, for round beams, for several given separations $\left(d_{x}, d_{y}\right)>0$. Figures 6 through 8 show pictures analogous to Figures 1 and 2, but now the separations are fixed and the amplitudes vary. The meaning of the contour plots is the same as before. If there is no contour plot shown, it means that the tune shift is positive everywhere.

Finally, it is worthwhile noting that the cutoff value $k=\max \left(\operatorname{nint}\left(a_{x}^{2} / 2\right), 1\right)$ is a good choice for the truncation of the infinite series for practical computations. The abbreviation nint stands for nearest integer. If the resulting tune shift is positive, the result of this truncation is a slight overestimation, while if the tune shift is negative the result is a slight underestimation of the tune shift with amplitude. If there are many long range interactions with different signs to sum, the errors almost cancel out each other. 

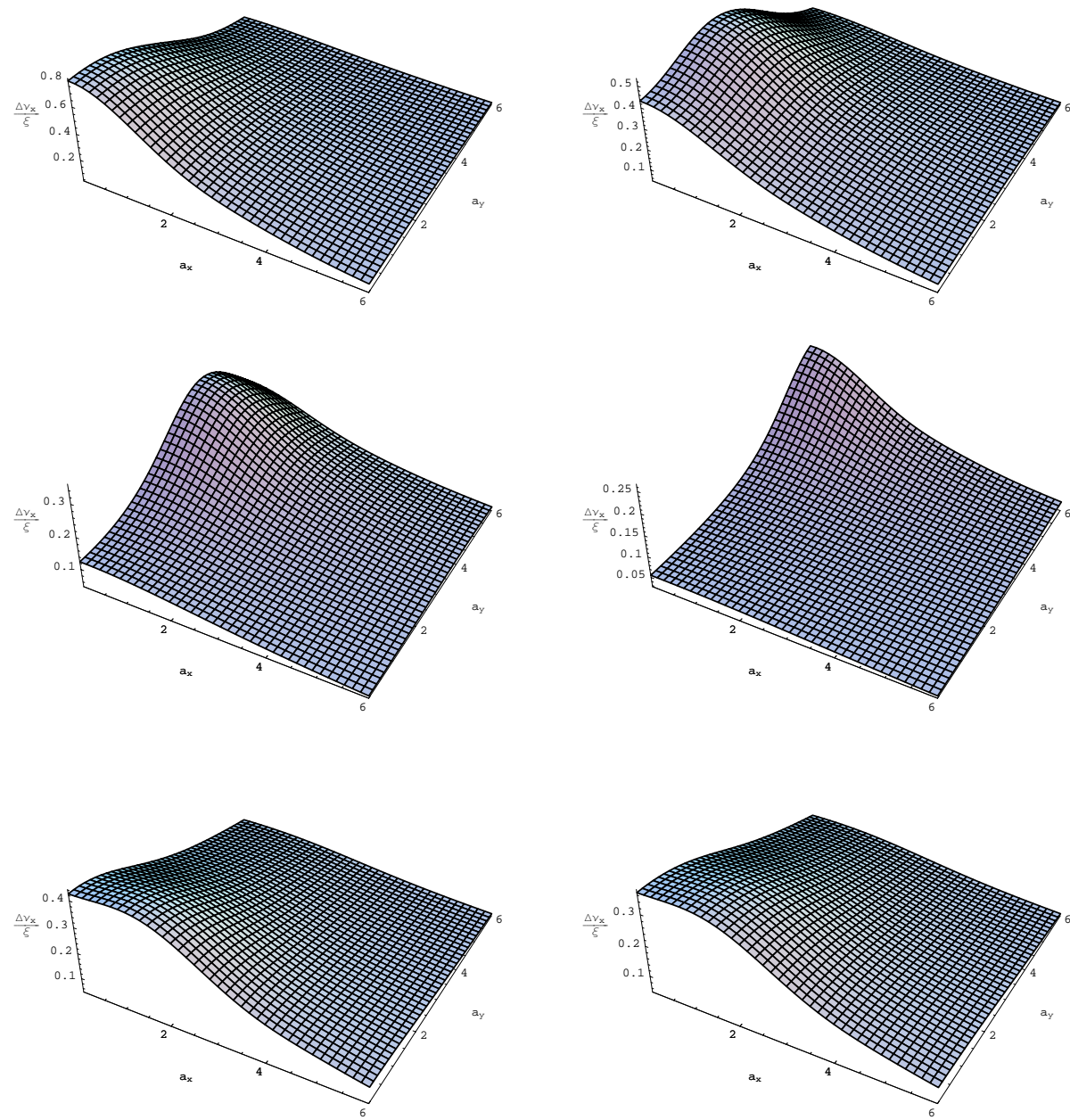

Figure 6: Horizontal tune shifts as a function of amplitudes, with $r=1$ and the following separations (increasing order of $\left.d_{x}\right):\left(d_{x}, d_{y}\right)=(0,1),(0,2),(0,4)$, $(0,6),(1,0)$, and $(1,1)$. 

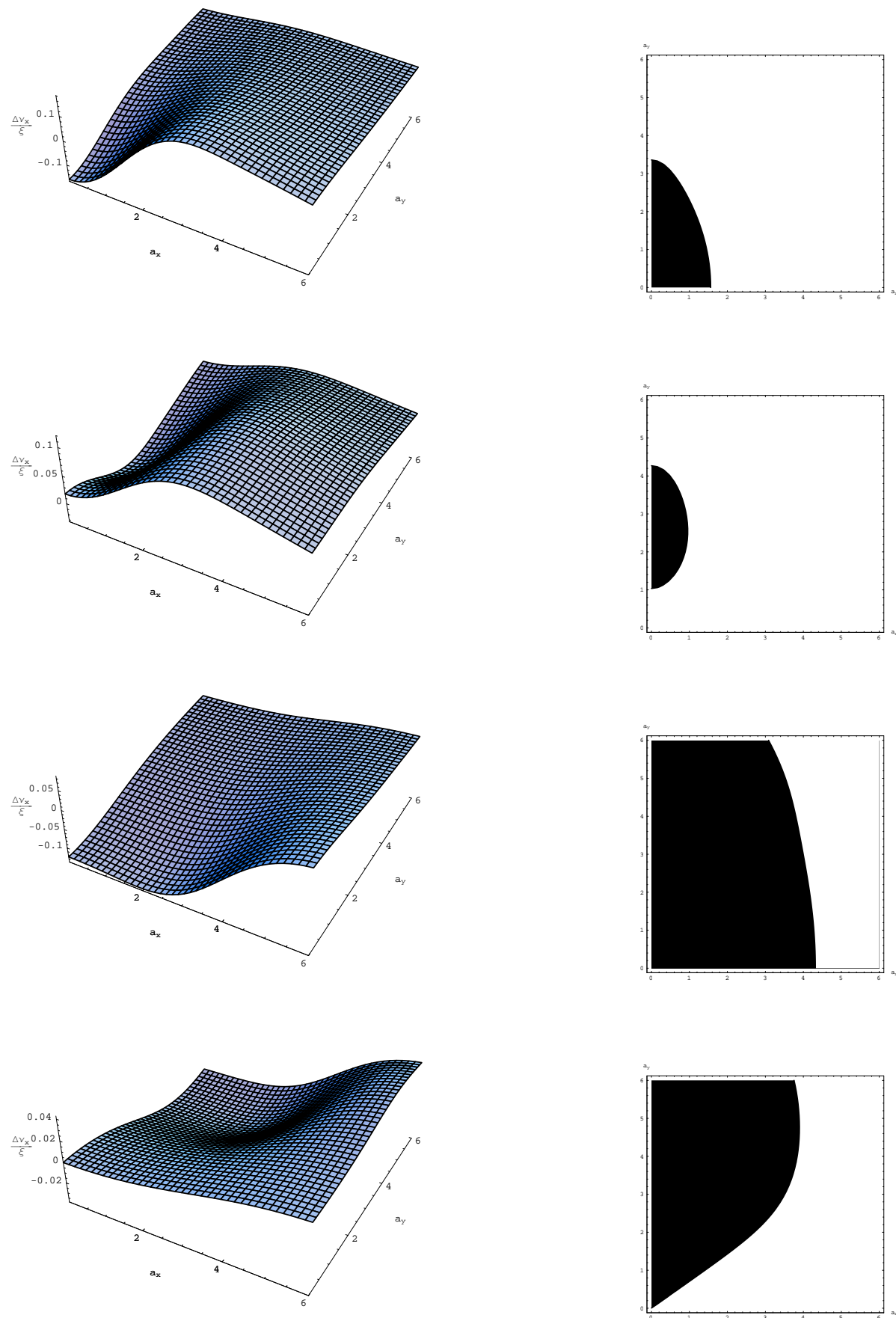

Figure 7: (Continuation of Figure 6) Horizontal tune shifts as a function of amplitudes, with $r=1$ and the following separations: $\left(d_{x}, d_{y}\right)=(2,0),(2,2)$, $(4,0)$, and $(4,4)$. 

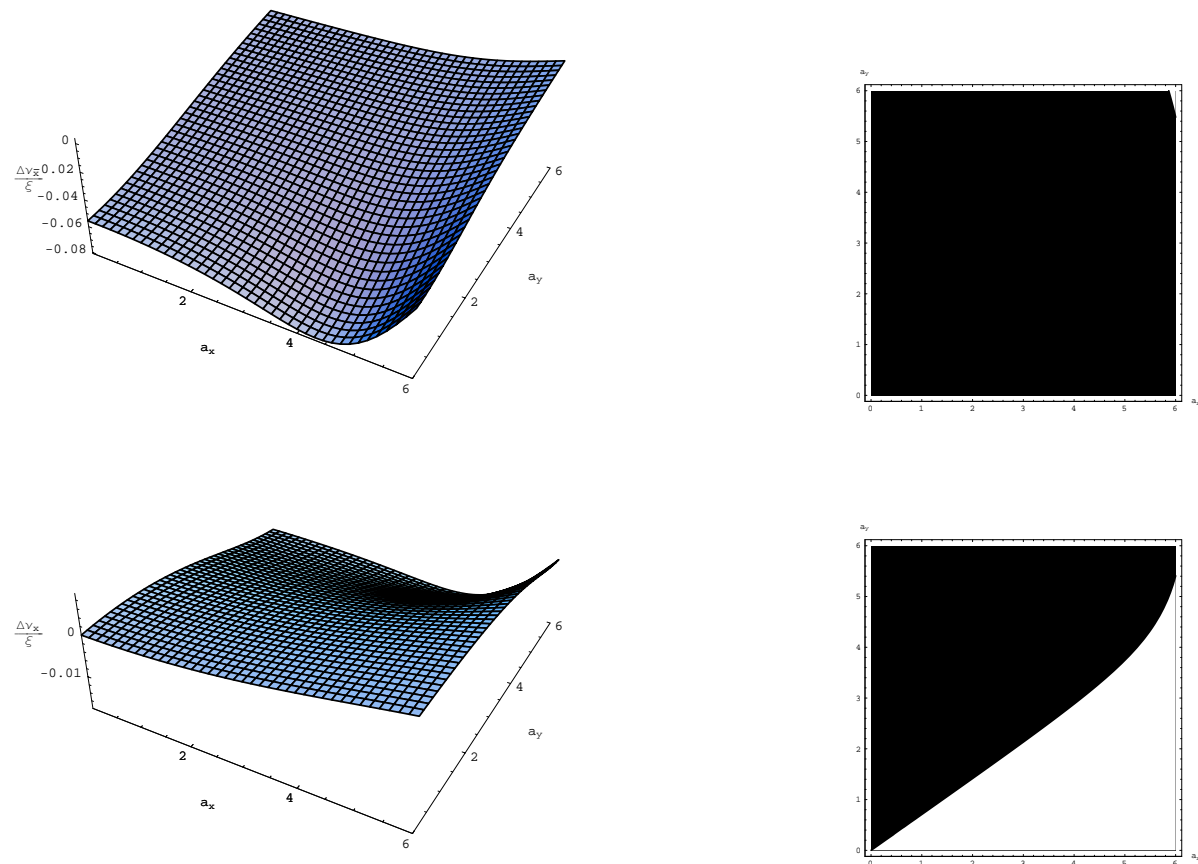

Figure 8: (Continuation of Figure 6) Horizontal tune shifts as a function of amplitudes, with $r=1$ and the following separations: $\left(d_{x}, d_{y}\right)=(6,0)$ and $(6,6)$. 


\section{Amplitude dependent chromaticity}

It is rather straightforward to include chromatic effects into (28), to provide a formula for the computation of the chromaticities. To this end, we split the separation into two parts: one due to the closed orbits of on-momentum particles, the other due to dispersion for off-momentum particles. Denoting the dispersion (in units of rms beam size) at the location of the interaction by $\eta$, first we make the following replacements in (28):

$$
\begin{aligned}
d_{x} & \mapsto d_{x}+\eta_{x} \delta, \\
d_{y} & \mapsto d_{y}+\eta_{y} \delta,
\end{aligned}
$$

where $\delta$ is the relative momentum or energy deviation. By definition, the linear chromaticities are given by

$$
Q_{x}^{\prime}=\left.\frac{\partial \Delta \nu_{x}}{\partial \delta}\right|_{\delta=0}, \quad Q_{y}^{\prime}=\left.\frac{\partial \Delta \nu_{y}}{\partial \delta}\right|_{\delta=0} .
$$

Unfortunately, the derivative of the tune shift with respect to momentum deviation is cumbersome and not very illuminating. However, using the symbolic capabilities of Mathematica, the derivative can be calculated symbolically, and then evaluated numerically. To this end, the horizontal chromaticity is given by

$$
\begin{aligned}
Q_{x}^{\prime}= & -\frac{2 \pi C}{a_{x} \varepsilon_{x}} \int_{0}^{1} \frac{e^{-\left(p_{x}+p_{y}\right)}}{\left[v\left(r^{2}-1\right)+1\right]^{1 / 2}} \times \\
& \sum_{k, l=0}^{\infty}\left[\frac{\Gamma\left(k+\frac{1}{2}\right) \Gamma\left(l+\frac{1}{2}\right)}{k ! l !}\left(\frac{a_{x}}{d_{x}}\right)^{k}\left(\frac{a_{y}}{d_{y}}\right)^{l}\left(A I_{l}\left(s_{y}\right)+B I_{l+1}\left(s_{y}\right)\right)\right] d v
\end{aligned}
$$

where

$$
\begin{aligned}
& A=2 d_{x}\left(d_{x} \eta_{x}+d_{y} \eta_{y} f\right) v I_{k-1}\left(s_{x}\right)-a_{x}\left(3 d_{x} \eta_{x}+2 d_{y} \eta_{y} f\right) v I_{k}\left(s_{x}\right)+ \\
& 2 \eta_{x}\left(a_{x}^{2} v-k-1\right) I_{k+1}\left(s_{x}\right)-s_{x} \eta_{x} I_{k+2}\left(s_{x}\right), \\
& B=2 a_{y} \eta_{y} f v\left(a_{x} I_{k}\left(s_{x}\right)-d_{x} I_{k-1}\left(s_{x}\right)\right) .
\end{aligned}
$$

The vertical chromaticity can be calculated similarly.

Note that the same cutoff values for $k$ and $l$ in the infinite series apply as for the tune shifts, since the convergence does not depend on the separation (i.e. dispersion). Despite the complicated structure of the formula, it can be seen that, of course, the chromaticity vanishes if there is no dispersion $\left(\eta_{x}=\eta_{y}=0\right)$, and also for head-on collisions, i.e.

$$
\lim _{d \rightarrow 0} Q_{x}^{\prime}=0,
$$

even for non-zero dispersions. Indeed, this follows from

$$
\lim _{z \rightarrow 0} \frac{I_{n}(z)}{z^{m}}=\left\{\begin{array}{c}
0, m<n, \\
\frac{1}{2^{n} n !}, m=n, \\
\infty, m>n,
\end{array}\right.
$$

as can be seen from the series representation of the Bessel functions (15). Using (67), it readily follows that none of the terms in (63) survive the limit of $d_{y} \rightarrow 0$ and $d_{x} \rightarrow 0$, and it gives identically zero linear chromaticity independent of the dispersions and amplitudes. 
On the other hand, for arbitrary dispersions and separations, but vanishing amplitudes, the following simplified formula can be obtained from (35):

$$
\begin{aligned}
\lim _{\substack{a_{x} \rightarrow 0 \\
a_{y} \rightarrow 0}} Q_{x}^{\prime}=\xi \int_{0}^{1} \frac{e^{-\frac{d_{x}^{2}+f d_{y}^{2}}{2} v}}{\left[v\left(r^{2}-1\right)+1\right]^{1 / 2}} v \times \\
\left(d_{x}^{3} \eta_{x} v+d_{x}^{2} d_{y} \eta_{y} f v-3 d_{x} \eta_{x}-d_{y} \eta_{y} f\right),
\end{aligned}
$$

which in the case of round beams gives the result

$$
\begin{aligned}
& q_{x}^{\prime}=\lim _{\substack{a_{x} \rightarrow 0 \\
a_{y} \rightarrow 0}} Q_{x}^{\prime}(r=1) \\
& =\xi \frac{2}{d^{6}}\left\{+e^{-\frac{d^{2}}{2}}\left[\begin{array}{c}
2\left(d_{x}^{3} \eta_{x}+3 d_{x}^{2} d_{y} \eta_{y}-3 d_{x} d_{y}^{2} \eta_{x}-d_{y}^{3} \eta_{y}\right) \\
-d_{x}^{7} \eta_{x}-d_{x}^{6} d_{y} \eta_{y}-d_{x}^{5}\left(2 d_{y}^{2}+1\right) \eta_{x}-d_{x}^{4}\left(2 d_{y}^{2}+3\right) d_{y} \eta_{y} \\
-d_{x}^{3}\left(d_{y}^{4}-2 d_{y}^{2}+2\right) \eta_{x}-d_{x}^{2}\left(d_{y}^{4}+2 d_{y}^{2}+6\right) d_{y} \eta_{y} \\
+3 d_{x}\left(d_{y}^{2}+2\right) d_{y}^{2} \eta_{x}+\left(d_{y}^{2}+2\right) d_{y}^{3} \eta_{y}
\end{array}\right]\right\} .
\end{aligned}
$$

Therefore, for large separations of the closed orbits, the zero amplitude chromaticity is

$$
q_{x}^{\prime}(\operatorname{large} d) \approx \xi \frac{4}{d^{6}}\left(d_{x} \eta_{x}\left(d_{x}^{2}-3 d_{y}^{2}\right)-d_{y} \eta_{y}\left(d_{y}^{2}-3 d_{x}^{2}\right)\right) .
$$

Notice that it decreases faster $\left(\sim 1 / d^{3}\right)$ with separation of the closed orbits than the corresponding tune shift. It is worthwhile to mention that along the diagonal in the $\left(d_{x}, d_{y}\right)$ plane, for large $d$, where the tune shift approximately vanishes for round beams, the corresponding chromaticity becomes

$$
q_{x}^{\prime}\left(\text { large } d_{x}=d y\right) \approx \xi \frac{4\left(\eta_{y}-\eta_{x}\right)}{\sqrt{2} d^{3}} .
$$

Therefore, it does vanish for equal dispersions in the two planes. More interestingly, for fixed separation $d$, the polar angle along which the chromaticity vanishes is given by solving (70) for the angle. One solution is given by

$$
\theta_{\min }=-\frac{1}{3} \operatorname{arccot}\left(\frac{\eta_{y}}{\eta_{x}}\right)
$$

The vertical dispersion is typically much smaller than the horizontal dispersion. In that case, when (70) is written in terms of the physical separations, then there are three possible solutions:

$$
\left(\frac{D_{y}}{D_{x}}\right)_{\min }=\left\{\begin{array}{c}
\frac{1}{\sqrt{3}}+\frac{4}{9} \lambda, \\
-\frac{1}{\sqrt{3}}+\frac{4}{9} \lambda, \\
-3 \frac{1}{\lambda}-\frac{8}{9} \lambda .
\end{array} \quad \lambda=\frac{\eta_{y}}{\eta_{x}}\right.
$$

The first two of these may be practically useful.

For fixed separation $d$ and dispersions, the angle at which the chromaticity vanishes varies with the aspect ratio. Again, it can be computed by numerical integration, and the results show two different qualitative behaviors, as a function of the dispersions' signs (same sign vs. opposite sign). In Figure 9 two representative cases are presented, using data taken from the Tevatron. In the case of same sign there is only one contour, while in the opposite sign case there are two contours along which the linear chromaticities vanish. In the latter case, the contour at smaller angles is always steeper than the contour at larger angles. 

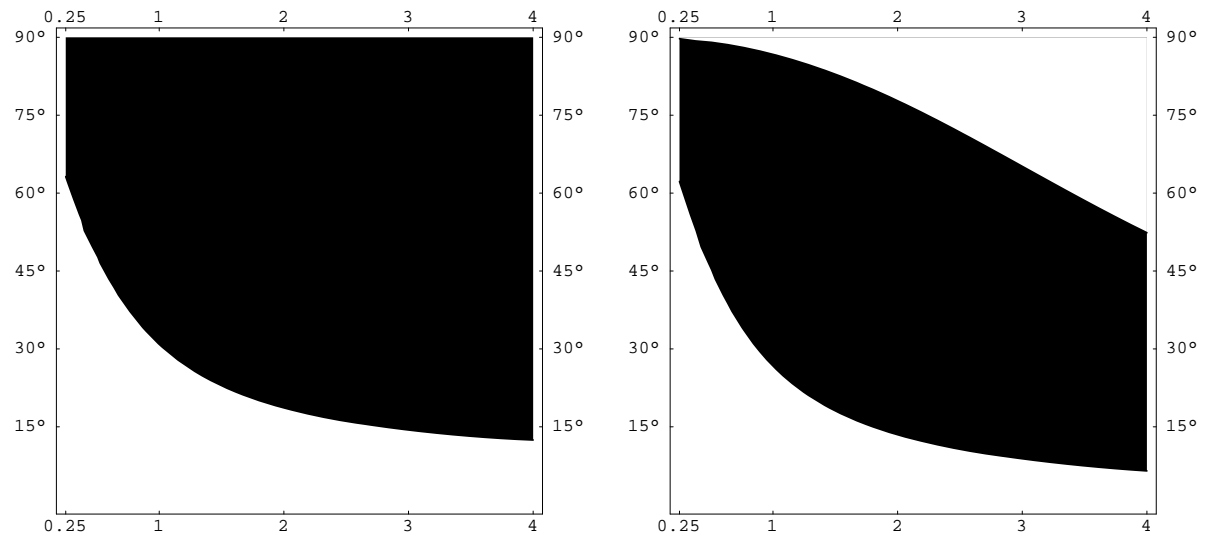

Figure 9: Contour of vanishing horizontal linear chromaticity at zero amplitude, $d=10$, and the following dispersions: $a$ ) average dispersions in the Tevatron (the horizontal and vertical dispersions have the same sign), and $b$ ) dispersion values at one of the nearest parasitics (\#55) (dispersions have opposite sign).

\section{Application to the Tevatron}

The tune shifts in the Tevatron are different from bunch to bunch [1]. For one $\bar{p}$ bunch (\# 6), using as separations the distances between the closed orbits at all the 72 encounters (shown in Figure 10), we computed the amplitude dependent tune shifts using (28) and (31) and summed up over all collisions. Inspection of the separations reveals that over the 70 long-range interactions $6 \lesssim|\vec{d}| \lesssim 14$ which entail tune shifts of different signs, according to (53) and (58). Computing the tune shifts at amplitudes of $\left(a_{x}, a_{y}\right)=(0,0),(0,6),(6,0)$, and $(6,6)$, we obtained the distribution depicted in Figures 11 (for the horizontal case) and 12 (for the vertical case). The distributions look similar for intermediate amplitudes. The maximum long range tune shifts are encountered at the interactions which are closest to the interaction points. Using tune shift information from intermediate amplitude values, we got the tune footprints shown in Figure 13. Overall, there is a good agreement with tracking results [1]; the maximum difference is about $10^{-3}$. As expected, the maximum value is obtained for zero amplitudes, and it tends to zero as the amplitude goes to infinity. The "length" of the footprint is given by the head-on interactions, while the main effect of the long range collisions is to widen the footprint at predominantly large horizontal and vertical amplitudes.

The scaling of the tune shifts and spreads with emittance have been assessed by computing the footprints at emittances that are $50 \%$ larger or smaller than the design emittance. The result is shown in Figure 14, where the resulting footprints are also superimposed on the footprint with the design emittance. Both the magnitude of the tune shifts and the size of the footprints increase with decreasing emittance. It means that the characteristics of the footprints are dominated by the head-on like interactions.

In order to explore possible correlations between folds in the tune footprint and dynamic aperture, we computed contour plots as functions of amplitude for three different cases: head-on and the four nearest parasitics, nearest parasitics only, and all collisions. Figures 15 through 16 contain the results. Notice that, in agreement with the analysis of section 3.2, folds appear at amplitudes of around $a_{x}, a_{y} \approx 7-8$ for the cases of parasitics only and all beam-beam. However, 


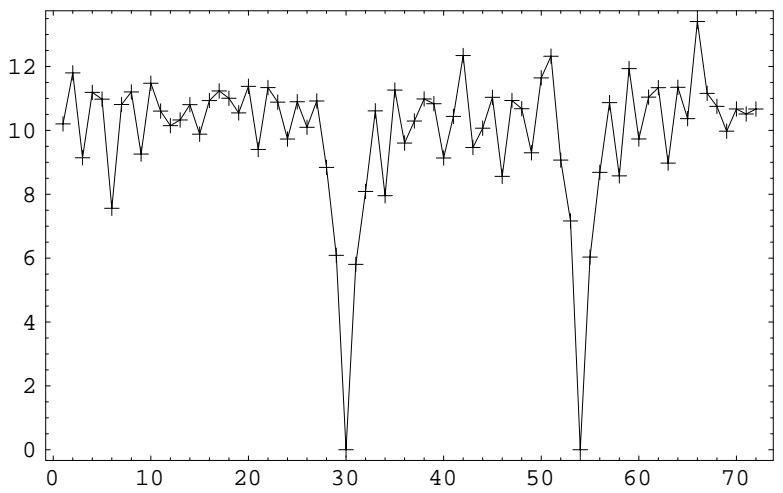

Figure 10: The separations $|\vec{d}|$ at all the 72 collisions between the antiproton bunch 6 and opposing proton bunches. head-on collisions occur at locations 30 and 54 .

there are no folds for the head-on and nearest parasitics case. This confirms the prediction that the folds are driven by the long range interactions and are counterbalanced by the head-ons. A sufficiently large number and strong long range collisions, which add up constructively, are necessary for the folds to form. However, tracking shows [1] that the nearest parasitics largely determine the 4D dynamic aperture, and the addition of the head-on collisions and/or the rest of the long range interactions do not alter significantly this DA. Therefore, from this point of view, the significance of the folds in the tune footprint is limited for Run IIa, although the location of the folds with all the beam-beam interactions is close to the dynamic aperture after $10^{5}$ turns. Nevertheless, there remains the possibility that the location of the folds in the nearest parasitics tune footprint is correlated with the dynamic aperture. Since without the head-ons the footprint is much smaller, it follows that the size of the footprint is also not correlated with the DA.

Since tracking shows that the nearest parasitics dominate the nonlinear dynamics, in the following we concentrate on these interactions only, and perform some studies aimed at unveiling correlations, if any, between this group of long range beam-beam interactions and the dynamic aperture.

The numerical experiments at the two different emittances mentioned above were repeated for the case of nearest parasitics only, to study the movement of the folds in amplitude space. Since the nearest parasitics dominate the size of the dynamic aperture, it is hoped that pushing the folds to larger amplitudes would have a beneficial effect on the dynamic aperture. The results presented in Figure 17 show that indeed the folds move in amplitude space as a function of emittance. More precisely, reduction of the emittance would result in pushing the folds to higher amplitudes. To obtain a more quantitative scaling law, we performed several searches for fold amplitudes at different emittances, both larger and smaller than the Tevatron RunIIa design emittance. The data points have been fitted to a curve; see Figure 18. We find that

$$
a(\text { fold }) \propto \frac{1}{\sqrt{\varepsilon}}
$$

This fact might provide an explanation for the difficulties the Tevatron is experiencing with long range beam-beam interactions at larger than design emittances. It is also worthwhile to note that, according to (40), the maximum values of the tune shifts along the folds vary with the emittance, with a fairly wide absolute maximum at a slightly smaller than the design emittance. 

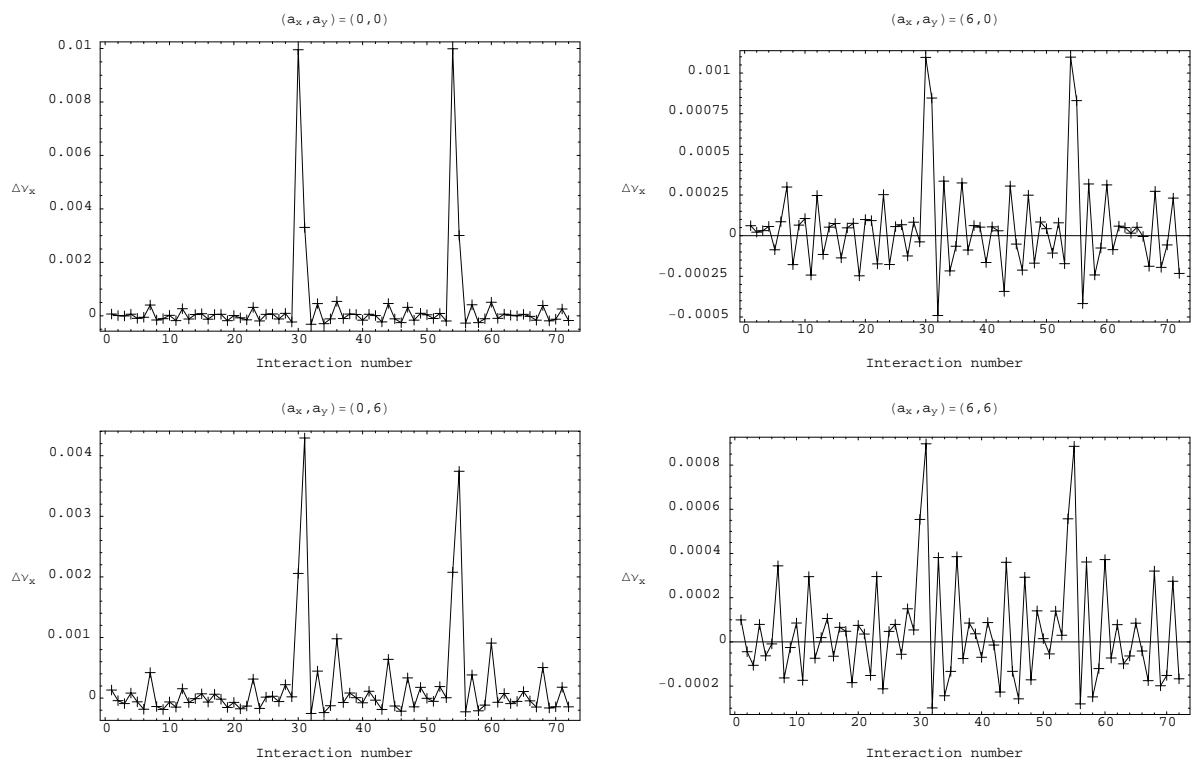

Figure 11: Horizontal amplitude dependent tune shifts for particles with the following amplitudes: $\left(a_{x}, a_{y}\right)=(0,0),(6,0),(0,6),(6,6)$. head-on collisions happen at interaction numbers 30 and 54 , while maximum long range tune shifts are at interaction numbers 55 and 31 .
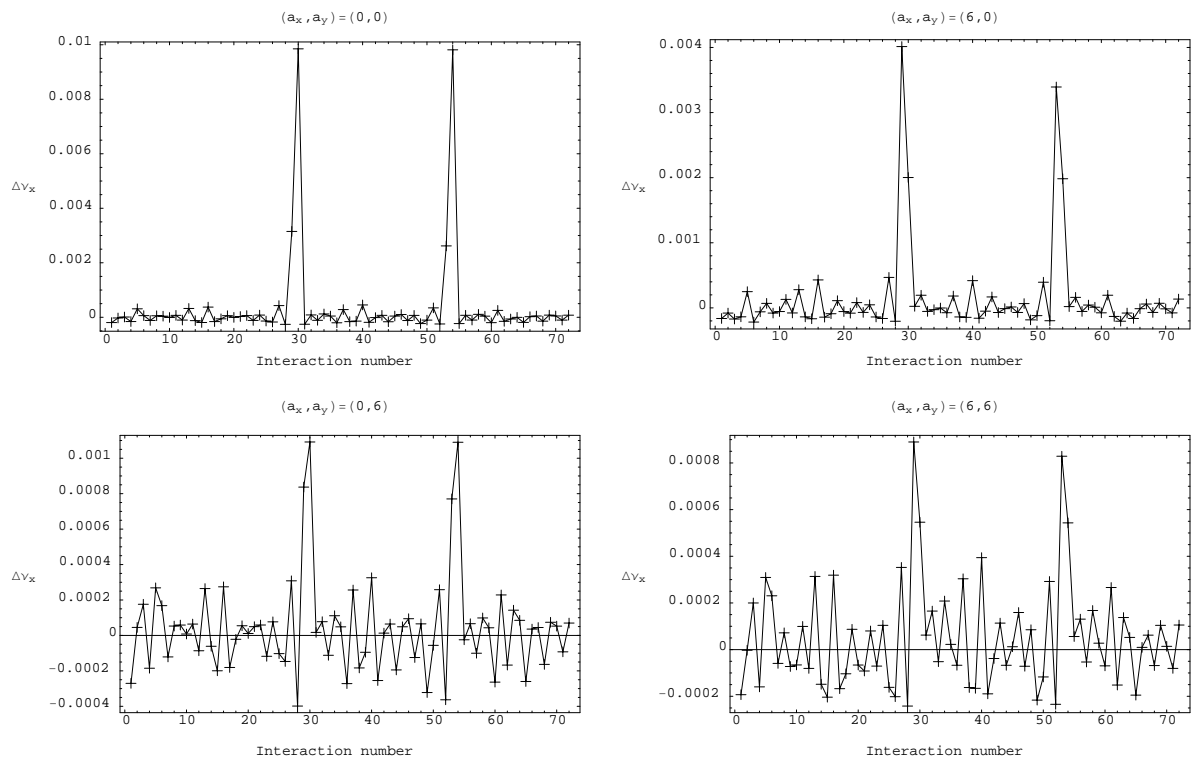

Figure 12: Vertical amplitude dependent tune shifts for particles with the following amplitudes: $\left(a_{x}, a_{y}\right)=(0,0),(6,0),(0,6),(6,6)$. head-on collisions happen at interaction numbers 30 and 54, while maximum long range tune shifts are at interaction numbers 29 and 53 . 

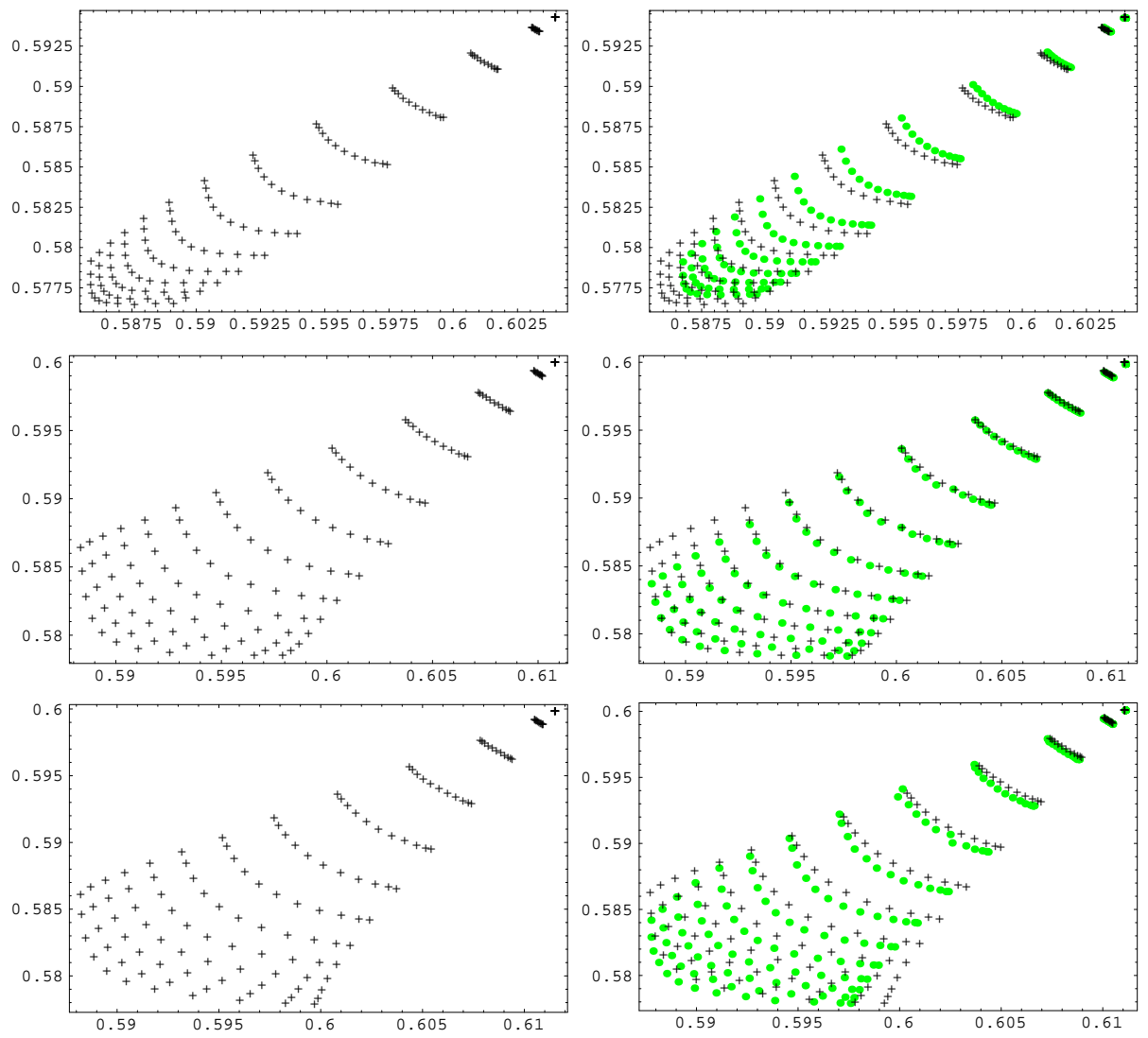

Figure 13: Tune footprints corresponding to the headon collisions only, to headon and nearest parasitic collisions, and to all 72 interactions. The left column was obtained from the analytical calculation, while the right column is a superposition of the left column with the tune footprints obtained by FFT of tracking data.
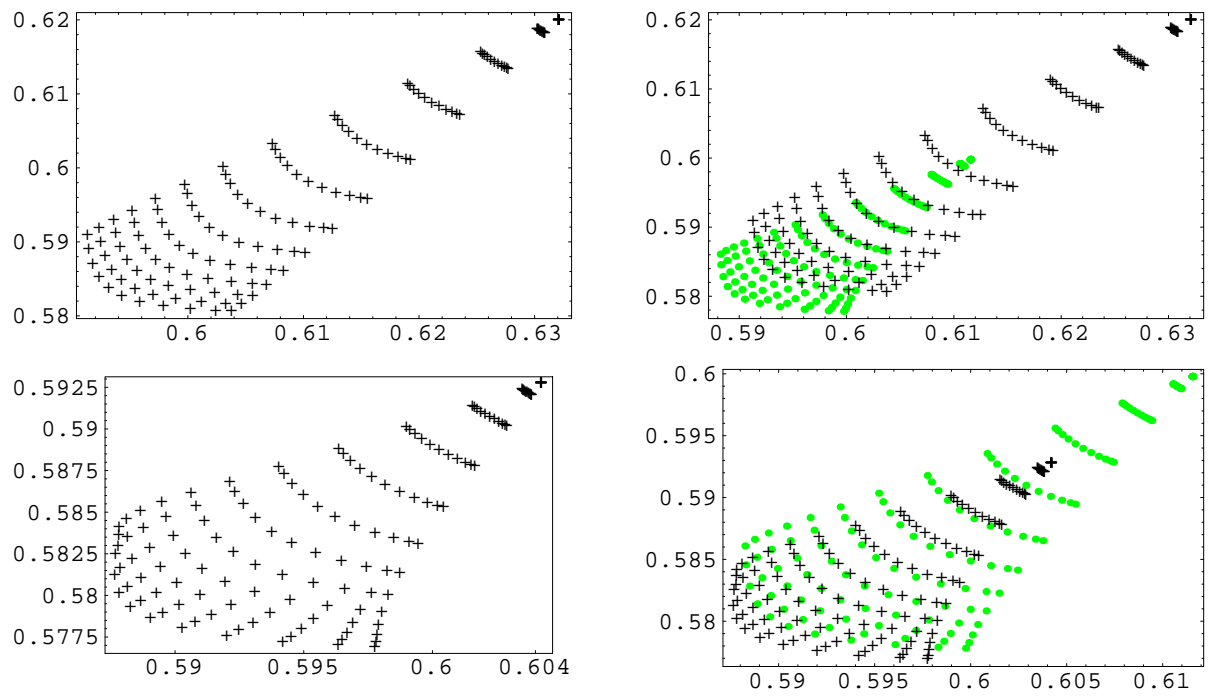

Figure 14: Left: Footprints at two different emittances: top figure - 50\% smaller, bootom figure - 50\% larger than the design emittance. Right: Superpositions of the footprints with the footprint obtained using the design emittance are shown. 
According to (66), the linear chromaticities induced by the head-on collisions are zero. The horizontal and vertical linear chromaticities induced by the four nearest parasitic interactions are displayed in Figure 19, where, as a function of equal transversal amplitudes $a_{x}=a_{y}$, the chromaticities are plotted for three different separations of the closed orbits, keeping the orientation of the separations in the $x-y$ plane unchanged. The figures show the same qualitative behavior, only their magnitude changes with separation, in the expected way. At amplitudes close to the dynamic aperture the chromaticities practically vanish, suggesting that the nearest parasitics induced chromatic effects might not be very important.

Nearest parasitic footprints were computed for both the tunes and the chromaticities. Figure 20 contains the results. Utilizing (43) and (72), an attempt was made in minimizing the footprints, by compensating for the aspect ratios or dispersions. Note that the compensating conditions were derived from the zero amplitude expressions of the corresponding relations, and hence it is not obvious a priori that the condition is useful for non-zero amplitudes, the case which is too cumbersome to treat it analytically. However, compensation of aspect ratios clearly reduces both the shift and the spread of the tunes, as can be seen in Figure 21. On the other hand, compensation of aspect ratios does not have a dramatic effect on the chromaticity footprint. Perhaps more importantly, aspect ratio compensation does not harm the chromaticity footprint, and in combination with the effect it has on the tune footprint, it might be useful in practice. The chromaticity footprint is mainly affected by compensation of the dispersions; there is a significant reduction in the size of the footprint. The results are contained in Figure 22.

A good check of potential correlations between these quantities and dynamic aperture is now possible by comparing the DAs in the following cases: design separations in the Tevatron, aspect ratio compensated nearest parasitics, and dispersion compensated nearest parasitics. The changes necessary in the lattice files are 8 numbers only: 4 horizontal and 4 vertical physical separations. Since the conditions specify the absolute values of the separations, the signs are arbitrary. It was found that keeping the original signs, and modifying only the magnitudes give the largest dynamic apertures. The tracking was performed with $\delta_{p}=3 \cdot 10^{-4}$, and otherwise similar conditions as in [1]. The results are presented in Table 1. It can be concluded that the compensations have a marginal effect on the DA, increasing the average DA by up to $\approx 0.5 \sigma$ and the minimum by $1 \sigma$, but fail to show the dramatic effects similar to the footprint size reduction. A probable cause of the poor performance of the dispersion compensation is that the chromaticity footprint with all the beam-beam interactions included is still relatively large when only the nearest parasitics are corrected. As shown in Figure 23, the rest of the parasitics induce large chromatic effects (see Figure $20 b$ for the uncorrected nearest parasitic chromaticity footprint). Therefore, in principle the total chromaticity footprint may be even more compressed if the compensation scheme is applied to all beam-beam interactions. Indeed, as also shown in Table 1 , in this case the DA is increased by the significant amount of almost $1 \sigma$, while the minimum DA stays unchanged. The total corrected chromaticity footprint fits into a 2 by 3 unit rectangle. Finally, we note that in the $4 D$ case, the aspect ratio compensation performs comparably with the $6 D$ case. 

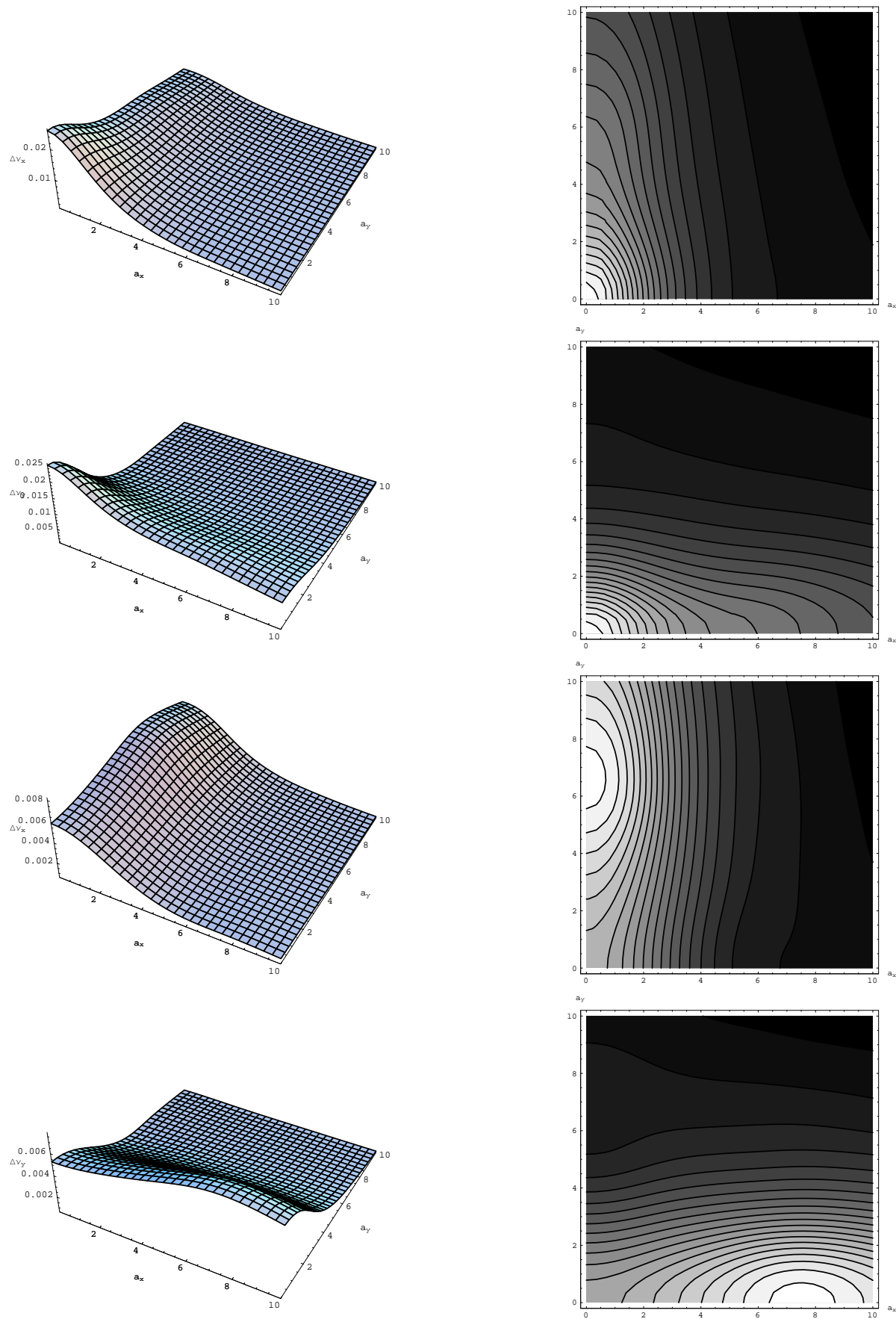

Figure 15: Top two plots (left and right): Horizontal and vertical amplitude dependent tune shifts (left) and corresponding contour plots (right) for the case of head-on and nearest parasitic collisions. No folds in the footprint are apparent. Bottom two plots (left and right): Horizontal and vertical amplitude dependent tune shifts and corresponding contour plots for the case of nearest parasitic collisions only. Folds in the footprint are apparent at amplitudes of $\left(a_{x}, a_{y}\right) \approx(8,7)$. 

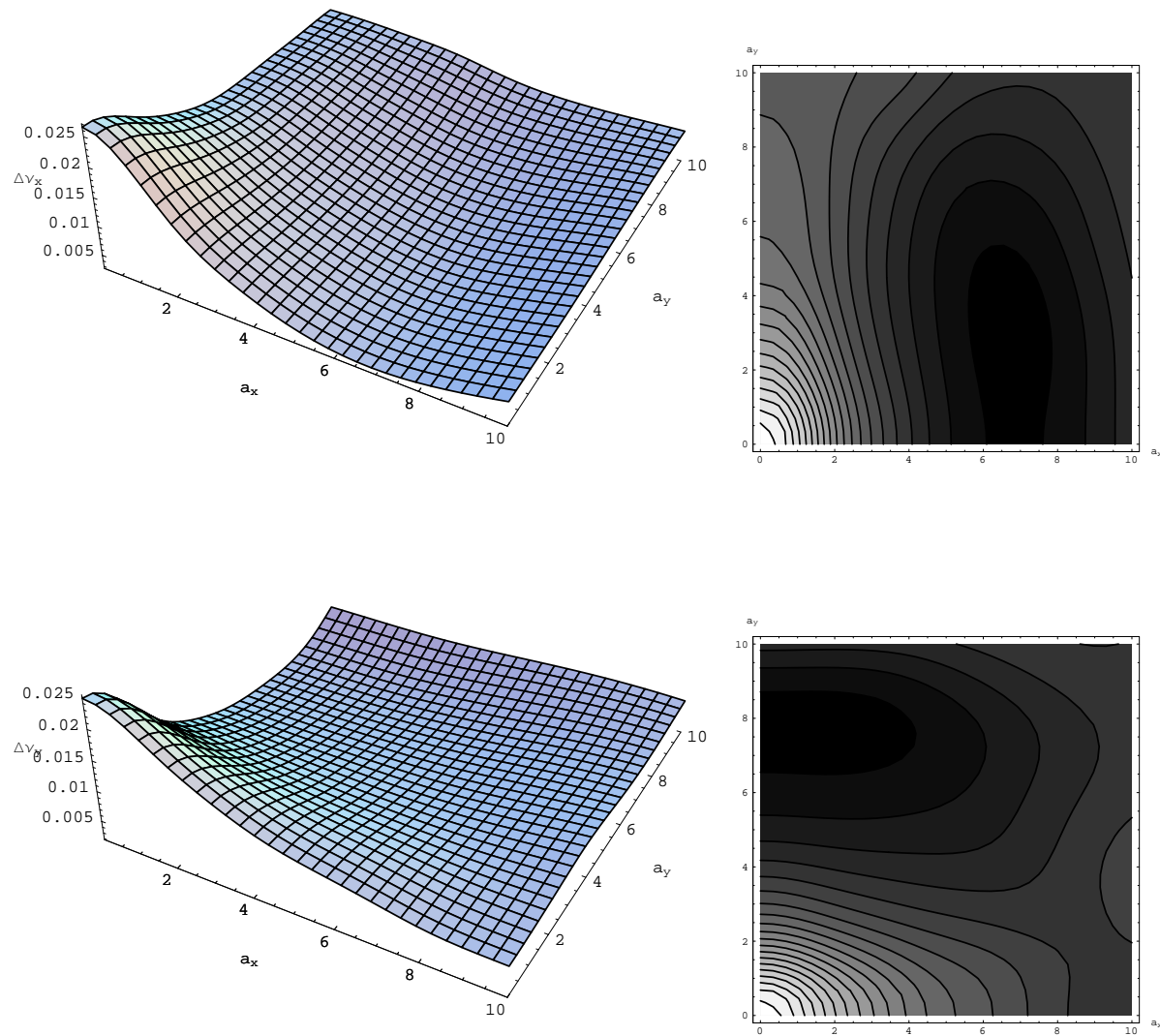

Figure 16: Horizontal and vertical amplitude dependent tune shifts and corresponding contour plots for the case of all beam-beam collisions. Although the folds have a different character than in the case of the nearest parasitics only, they do appear in the footprint at approximately the same amplitudes of $\left(a_{x}, a_{y}\right) \approx(7,8)$, close to the dynamic aperture.

\begin{tabular}{|c|c|c|}
\hline Case & $\langle$ DA $\rangle$ & DA $_{\min }$ \\
\hline Original Tevatron RunIIa configuration & 7.4 & 6 \\
\hline Aspect ratio compensated tune and chrom. footprint & 8.0 & 7 \\
\hline Dispersion compensated chromaticity footprint for: & & \\
Nearest parasitics only & 7.7 & 7 \\
All beam-beam interactions & 8.3 & 6 \\
\hline
\end{tabular}

Table 1: The effects of the footprint compensation schemes on the dynamic aperture. The $6 \mathrm{D}$ dynamic aperture was computed after 100,000 turns. 

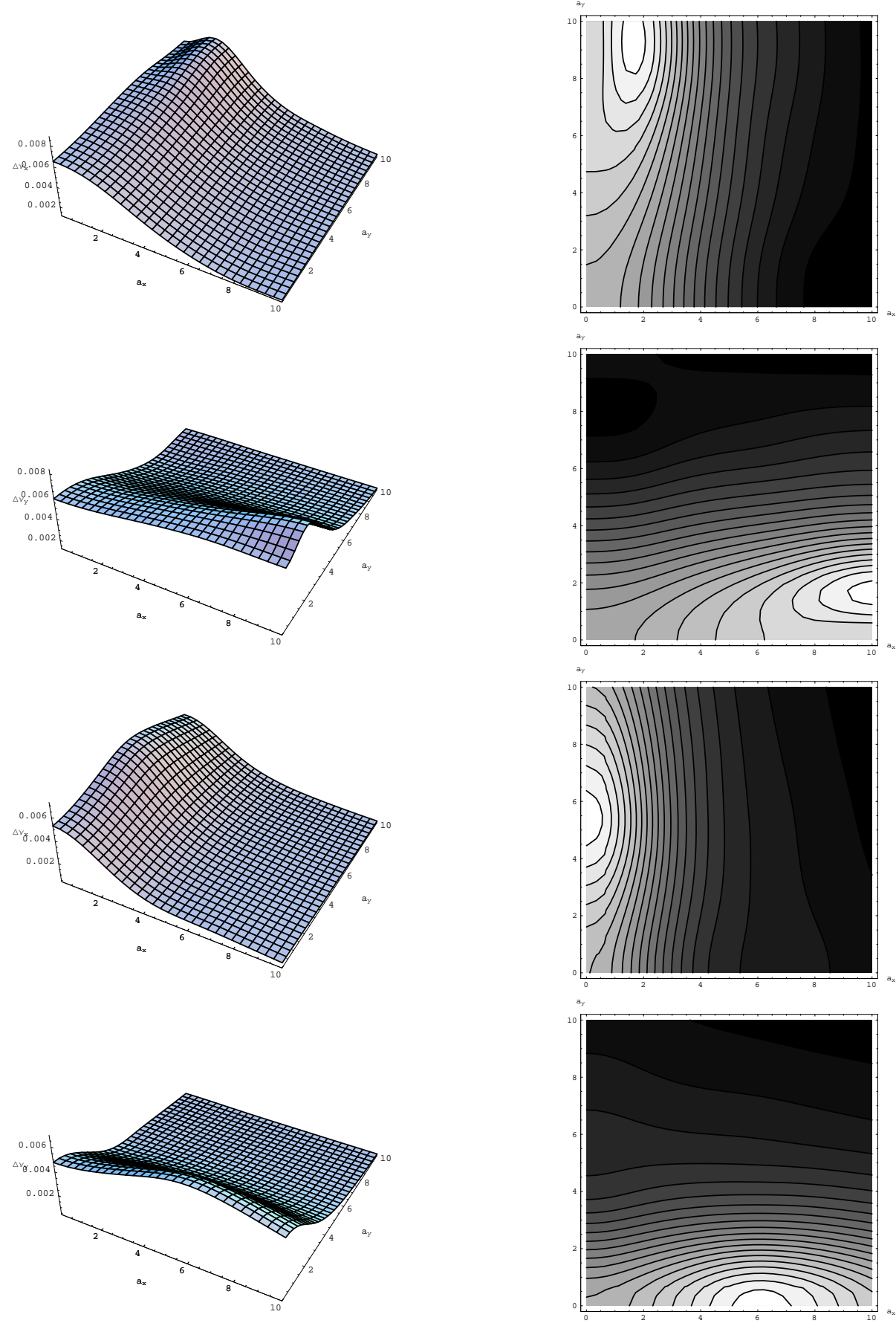

Figure 17: Top two plots (left and right): Location of the horizontal and vertical folds in amplitude space and contour plots, computed at 50\% smaller emittance than the design emittance. Bottom two plots (left and right): Location of the horizontal and vertical folds in amplitude space, computed at 50\% larger emittance than the design emittance. 


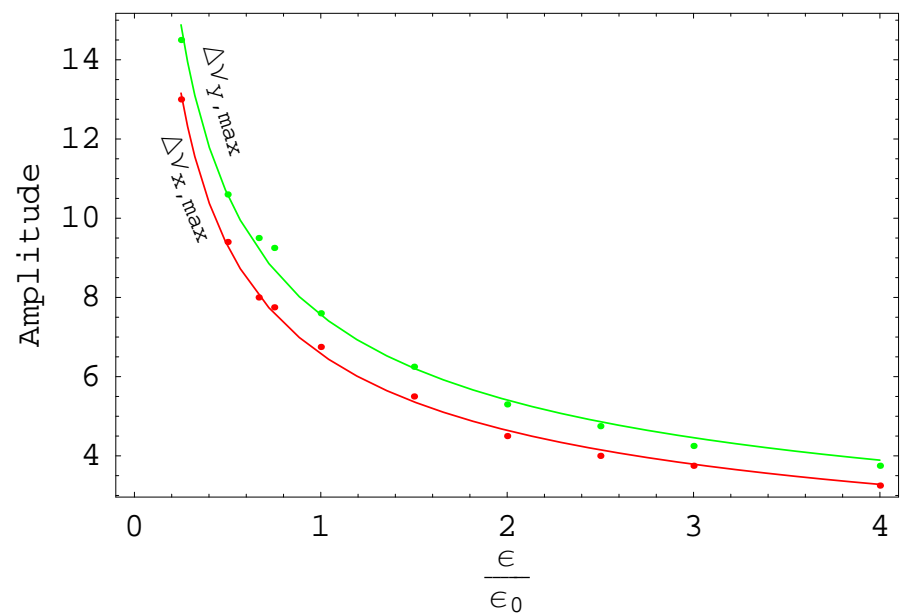

Figure 18: Inverse square root fit of the location of fold amplitudes as a function of emittance, when only the nearest parasitics are included in the computation.
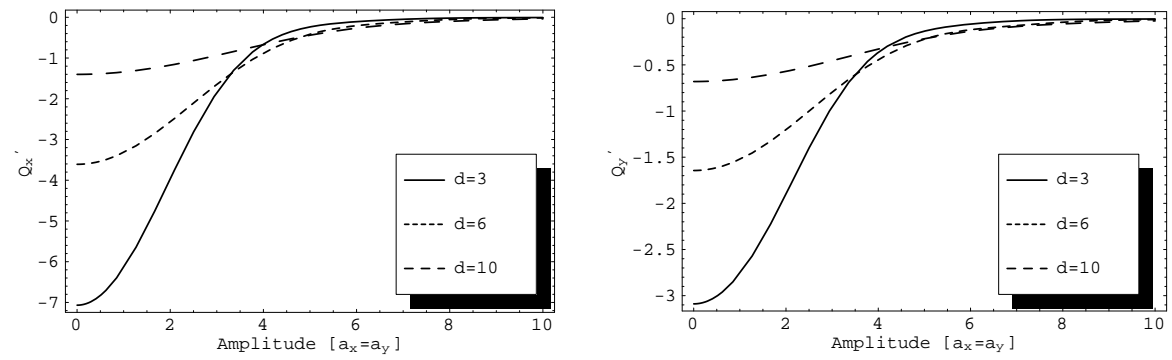

Figure 19: Horizontal and vertical amplitude dependent linear chromaticities for the case of the nearest parasitic collisions at three different separations of the closed orbits.
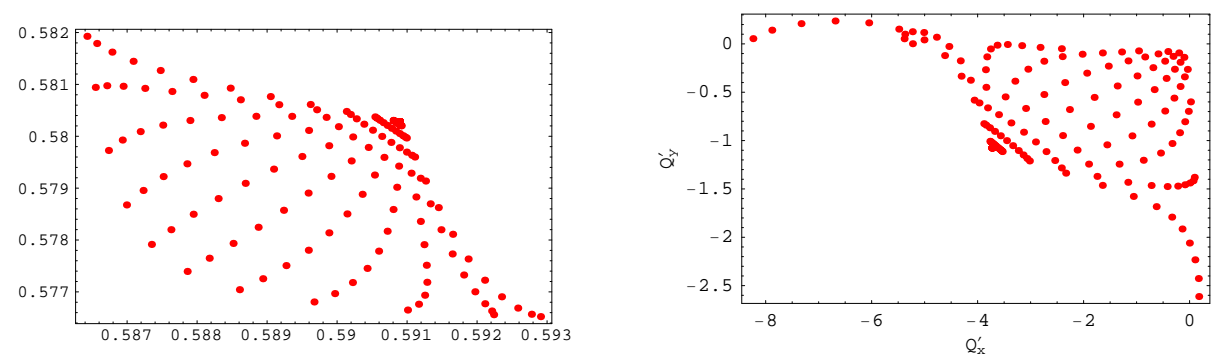

Figure 20: $a$ ) Tune and $b$ ) chromaticity footprints of the nearest parasitic beambeam interactions in the original Tevatron RunIIa configuration for antiproton bunch \#6. 


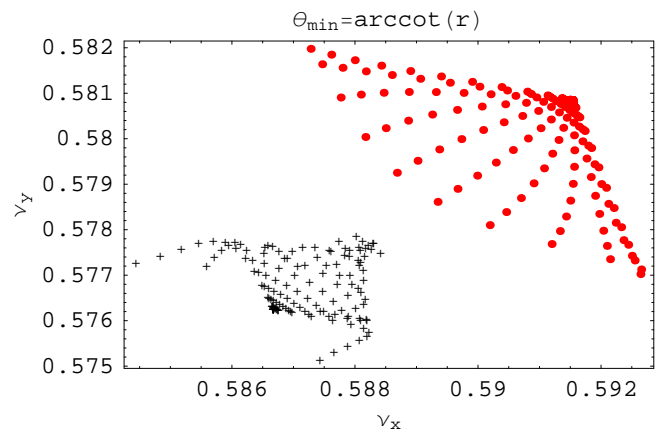

Figure 21: Tune footprint compensation of the nearest parasitic beam-beam interactions (shown in Figure 20a), by adjusting the physical separations in such a way that the distances and the signs stay the same, and only the magnitudes of $D_{x}$ and $D_{y}$ are modified, by the relation $\left|D_{x}\right|=\left|D_{y}\right|$, keeping $D_{x}^{2}+D_{y}^{2}$ the same. Here, and in the following three figures, dots represent the footprint before correction, while the ' + ' symbols represent the footprint after correction.
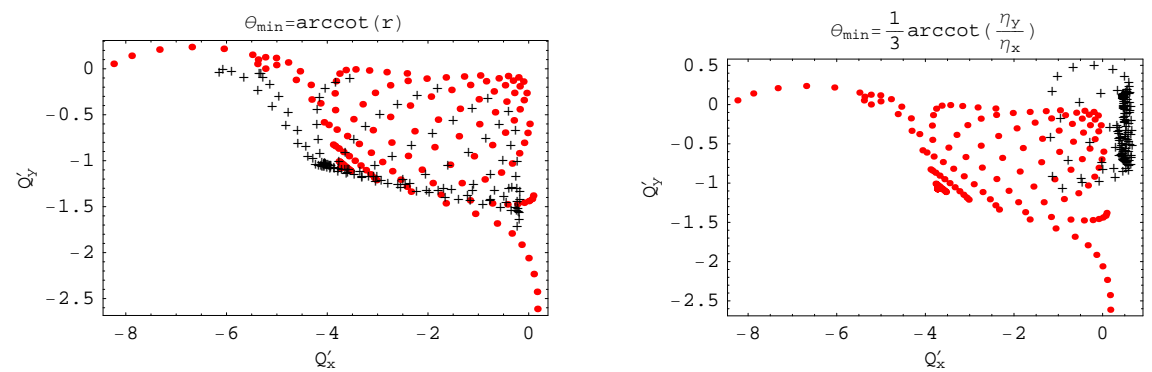

Figure 22: Chromaticity footprint compensation of the nearest parasitic beambeam interactions (shown in Figure 20a), by a) the same method as in Figure 21, and $b$ ) adjusting the physical separations in such a way that the distances and the signs stay the same, and only the magnitudes of $D_{x}$ and $D_{y}$ are modified, by equation (72).
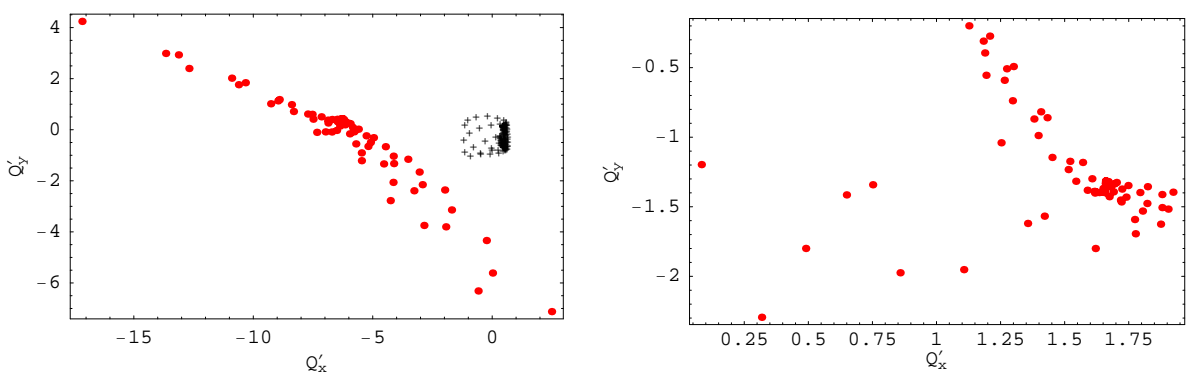

Figure 23: Left: Comparison of two chromaticity footprints: (i) All beam-beam interactions included, no compensation. The spread in chromaticities spans about 20 units horizontally and 11 units vertically. (ii) Nearest parasitics only but compensated with dispersion correction. The spread in chromaticities spans only about 2 units in each plane. Right: The footprint from all interactions compensated for the dispersion at all interactions. This footprint now spans $(2$, 3) units in the two planes. 


\section{Conclusions}

We derived a useful analytical tool for the computation of the amplitude dependent tune shifts and linear chromaticities due to beam-beam interactions. The expressions can be used for efficient numerical evaluation at any amplitude, separation, dispersion, and aspect ratio. The favorable convergence properties make it especially suitable for studies of the parasitic beam-beam interactions. The structure of the equations is well suited for studying sign changes (locations of folds in the footprint), maxima and/or minima, and scalings of the tune shifts and chromaticities with respect to beam parameters such as emittances.

These expressions are in general complicated but simplify when evaluated for round beams and at zero betatron amplitude. The dominant contribution to the zero amplitude tune shifts falls off as the inverse square of the beam separation while the dominant contribution to the zero amplitude chromaticity falls off as the inverse cube of the beam separation.

Applied to the Tevatron, we learned that, in general, keeping everything symmetric helps in minimizing tune shifts and chromaticities. For example, if the physical separation is along the diagonal in the $(x, y)$ plane, the long range zero amplitude tune shift (approximately) vanishes at reasonably large separations. If, additionally the dispersions are also equal, the linear chromaticity vanishes too. In the more typical case where the vertical dispersion is much smaller than the horizontal dispersion, the ratio of physical separations which minimizes the chromaticity is determined by the aspect ratio and the ratio of the dispersions (see Equation (73) ).

We found that if the separations are chosen to minimize either the zero amplitude tune shift or chromaticity, then the tune shifts and chromaticities at non-zero amplitudes are significantly smaller as well.

The scaling of the long range tune shift with emittance is qualitatively different from the head-on case. At least for vanishing amplitudes, the absolute value of the long range tune shift has a maximum at a certain emittance, and decreases for smaller emittances, contrary to the head-on tune shift, which always scales like $1 / \varepsilon$. We also studied the location of the folds in the tune footprint due to the parasitics nearest to the IPs as a function of emittance. We found that the amplitude at the folds scales as $1 / \sqrt{\varepsilon}$. This implies that at larger emittances, the effects of resonances closer to the beam core will be enhanced if they coincide with the location of the folds.

We examined the impact of reducing the tune footprint on the dynamic aperture. The nearest parasitics in the Tevatron dominate the contributions of all the parasitics to the tune footprint. Separations at the nearest parasitics were adjusted to minimize the footprint. This reduced the tune footprint substantially and also reduced the chromaticity footprint slightly. The dynamic aperture was found to increase by about $0.6 \sigma$. This reinforces the conclusion reached in [1] that the correlation between the size of the tune footprint and the dynamic aperture is at best indirect.

Finally we examined the impact of reducing the chromaticity footprint. The nearest parasitics do not dominate the contributions to this footprint. When only the separations at the nearest parasitics were adjusted to minimize their contribution, there was a negligible increase in dynamic aperture. However, when the separations at all the parasitics were adjusted to minimize the chromaticity footprint, then the dynamic aperture increased by about $1 \sigma$ which is significant. While this compensation scheme is not practical, due to the constraints on the Tevatron optics, it does point to mechanisms which may increase the stable area available to the beam. For example, reducing the momentum spread in the beam and the linear chromaticity in the Tevatron at top energy 
would be helpful.

\section{References}

[1] T. Sen, M. Xiao. Beam-Beam Interactions in Run IIa. FNAL Preprint, to be published, Fermilab, Batavia, 2002.

[2] M. Berz. Differential Algebraic Formulation of Normal Form Theory. In $M$. Berz, S. Martin and K. Ziegler (Eds.), Proc. Nonlinear Effects in Accelerators, page 77, London, 1992. IOP Publishing.

[3] M. Berz, B. Erdelyi, W. Wan, and K. Ng. Differential Algebraic Determination of High-Order Off-Energy Closed Orbits, Chromaticities, and Momentum Compactions. Nuclear Instruments and Methods, A427:310-314, 1999.

[4] M. Bassetti and G.A. Erskine. CERN-ISR-TH/80-06. 1980.

[5] D. Neuffer and S. Peggs. Beam-Beam Tune Shifts and Spreads in the SSC Head-On, Long Range, and PACMAN Conditions. Technical Report SSC63, SSC Central Design Group, 1986.

[6] G.N. Watson. A Treatise on the Theory of Bessel Functions. Cambridge University Press, 1966.

\section{A Alternate Derivation of a Formula for the Am- plitude Dependent Tune Shifts}

There is an alternate way to derive a formula for analytical evaluation of the amplitude dependent tune shift of long range beam-beam interactions. It uses the generating function expression of the modified Bessel function of the first kind

$$
e^{-z \cos \theta}=\sum_{k=-\infty}^{\infty}(-1)^{k} I_{k}(z) \cos k \theta
$$

With the slightly different shorthand notations

$$
\begin{aligned}
p_{x} & =\frac{v}{2}\left(\frac{a_{x}^{2}}{2}+d_{x}^{2}\right), \\
r_{x} & =\frac{v}{4} a_{x}^{2}, \\
s_{x} & =v a_{x} d_{x},
\end{aligned}
$$

and similarly for the $y$ case, the following relation is obtained:

$$
\Delta \nu_{x}=-\frac{N_{b} r_{p}}{4 \pi \gamma_{p} \varepsilon_{x}} \int_{0}^{1} \frac{e^{-\left(p_{x}+p_{y}\right)}}{\left[v\left(r^{2}-1\right)+1\right]^{1 / 2}} F_{x} F_{y} d v,
$$

where

$$
\begin{aligned}
& F_{x}=\sum_{k=-\infty}^{\infty}(-1)^{k}\left\{\left[I_{k}^{\prime}\left(r_{x}\right)-I_{k}\left(r_{x}\right)\right] I_{2 k}\left(s_{x}\right)+\frac{2 d_{x}}{a_{x}} I_{k}\left(r_{x}\right) I_{2 k}^{\prime}\left(s_{x}\right)\right\}, \\
& F_{y}=\sum_{l=-\infty}^{\infty}(-1)^{l} I_{l}\left(r_{y}\right) I_{2 l}\left(s_{y}\right),
\end{aligned}
$$


The rest of the symbols have the same meaning as before. A similar relation is obtained for the vertical tune shift.

The infinite series in the tune shift's expression does converge (the error is on the order of magnitude of the first neglected term), but, due to the alternating nature of the series, it is prone to numerical inaccuracies due to cancellation of digits (occurrence of large terms of the same order of magnitude but different signs), especially at large separations. That is why (28) is better suited for the long range case. Moreover, it is not clear if an analysis similar to section 3 can be carried out.

We conclude the paper with an interesting connection between this form of the formula for the amplitude dependent tune shift computation and the original, (28). As shown in section 2, the sum

$$
S_{1}=\sum_{k=0}^{\infty} \frac{\left(\frac{a_{x}}{d_{x}}\right)}{k !} \Gamma\left(k+\frac{1}{2}\right) I_{k}\left(s_{x}\right)
$$

has a rate of convergence $R_{n}$ satisfying

$$
R_{n} \leq a_{x}^{2} v \frac{2 n+1}{4(n+1)^{2}} .
$$

If the equal sign is assumed in (83), which is valid for small $s_{x}$, the following upper bound is obtained for $S_{1}$ :

$$
\begin{aligned}
S_{1} & \leq S_{2}=\sum_{k=0}^{\infty} A_{k}=A_{0}+A_{1}+A_{2}+\ldots \\
& =A_{0}+A_{0} R_{0}+A_{1} R_{1}+\ldots \\
& =A_{0}\left(1+R_{0}+R_{1} R_{0}+R_{2} R_{1} R_{0}+\ldots\right) \\
& =A_{0}\left(1+\sum_{k=1}^{\infty} \prod_{n=0}^{k-1} R_{n}\right) .
\end{aligned}
$$

This expression can be summed up knowing that

$$
\prod_{n=0}^{k-1} R_{n}=\left(\frac{a_{x}^{2} v}{2}\right)^{k} \frac{\Gamma\left(k+\frac{1}{2}\right)}{\sqrt{\pi}(k !)^{2}} .
$$

Combining this with

$$
A_{0}=\sqrt{\pi} I_{0}\left(s_{x}\right),
$$

and utilizing the notation of this section we obtain for the infinite sum that

$$
S_{2}=\sqrt{\pi} e^{r_{x}} I_{0}\left(r_{x}\right) I_{0}\left(s_{x}\right) .
$$

Comparing (28) with (79), it follows that, using $S_{2}$ for the infinite sum, the approximation gives the same result with the truncation $k=l=0$ in (79). Therefore, the small amplitude and separation case is well represented by a single term from the infinite sums, and from $S_{2}$ being an upper bound it follows that

$$
\sum_{k=1}^{\infty}(-1)^{k} I_{k}\left(r_{x}\right) I_{2 k}\left(s_{x}\right) \leq 0
$$

for any amplitude and separation. 